\title{
Algorithms for Fixture Design
}

\author{
Chantal Wentink* A. Frank van der Stappen* Mark Overmars*
}

\begin{abstract}
Manufacturing and assembly processes often require objects to be held in such a way that they can resist all external wrenches. The problem of "fixture planning" is to compute, for a given object and a set of fixturing elements, the set of placements of the fixturing elements that constrain all finite and infinitesimal motions of the object (due to applied wrenches). As fixturing problems occur frequently in manufacturing and assembly, it becomes costly to build a dedicated fixturing solution for each different problem. Modular fixturing toolkits offer the advantage of reusability of the fixturing elements and have therefore gained considerable popularity. A modular fixturing toolkit consists of a fixturing table with a rectangular grid of holes, and a set of fixturing elements whose positions are restricted to the holes in the table. Several recent publications in the field of fixture planning aim at exploring the power of these modular fixturing toolkits. We give an overview of modular and non-modular fixture planning for various types of objects and sets of fixturing elements.
\end{abstract}

\section{Introduction}

Many manufacturing operations, such as machining, assembly, and inspection, require constraints on the motions of parts or subassemblies of parts $[4,8]$. The concept of form-closure is over a century old [25] and refers to constraining, despite the application of an external wrench (force and moment), all motions of a rigid object (including infinitesimal motions) by a set of contacts on the object; any motion of an object in form-closure has to violate the rigidity of the contacts. Therefore, the problem is to compute contact locations on a given part shape that achieve form-closure.

In this paper, we are interested in immobilizing planar objects, in particular polygons. We give an overview of fixturability results and algorithms to compute form closure configurations under different fixture models. We refer to the set of contacts achieving form-closure as a fixture. We assume that the contacts are frictionless; note that this is a conservative assumption since any fixture computed assuming zero friction also holds in presence of friction. By the fixture model, we imply the set of allowable contact types. The conceptually simplest model is that of point contacts. See Figure 1 for an example of a fixture

\footnotetext{
${ }^{*}$ Department of Computer Science, Utrecht University, P. O. Box 80.089, 3508 TB Utrecht, the Netherlands, Email: \{chantal, frankst, markov\}@cs.ruu.nl. This work was partially supported by the Netherlands Organization for Scientific Research (N.W.O.) and by a NATO collaborative research grant number CRG.951224.
} 


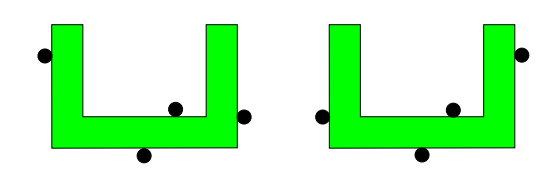

Figure 1: A polygon that is not fixtured with four point-contacts and the same polygon that is fixtured.

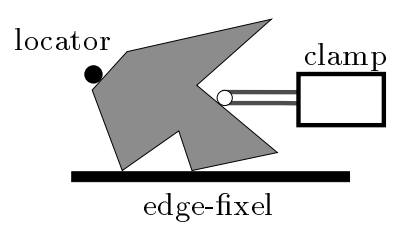

Figure 2: A polygon in form closure with one edge-contact and two pointcontacts. A point-fixel can reach into concavities, whereas an edge-fixel cannot.

in a model with four point-contacts. It has been known since Reuleaux that fixturing a planar object requires at least four frictionless contacts. Mishra, Schwartz, and Sharir [21] and Markenscoff, Ni and Papadimitriou [19] independently proved that four point-contacts are also sufficient.

While point-contacts are conceptually simple, they are not always easy to achieve in practice. The reason is that for form-closure, these point-contacts have to be capable of resisting arbitrary wrenches and therefore they have to be backed by bulky supports. This in turn implies difficulty of placing pointcontacts at some points on the boundary of an object, in particular, at narrow concavaties. Hence, it is important to look for other possible practical fixture models in order to reduce the number of point-contacts. In everyday life, we frequently lean an object against a flat surface, such as a table or a wall, to constrain its motions. In the planar world, the analog of a wall is a supporting line. In this paper we also consider fixture models that include edge-contacts which offer straight-lines of support. Notice that an edge-contact can touch the object only along its convex hull. The object simply rests against it; there is no reaching into concavaties. See for an example Figure 2.

The first part of this overview is concerned with fixturability results. We identify classes of parts that are fixturable under different fixture models including point-contacts and one or two edge-contacts. Since it is not only important to know what parts can be fixtured under a model, but also what parts can not be fixtured, we also give negative results on existence of solutions.

The second part of the paper deals with modular fixtures which is a subject of considerable popular interest in the manufacturing industry for the past ten years or so $[1,2,13,14]$. Basically, this involves a regular square grid of lattice holes together with fixture elements (or fixels [5]) that are constrained by the grid; the object rests against these fixels which constrain its motions. Custombuilt fixtures being expensive, the major benefit of modular fixtures stems from 


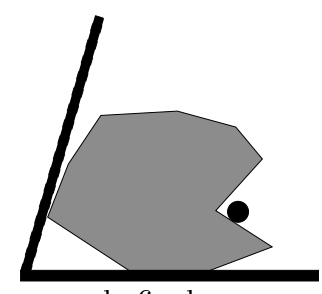

angle-fixel

Figure 3: A polygon in form closure with two edge-fixels (one angle-fixel) and a point-contact.

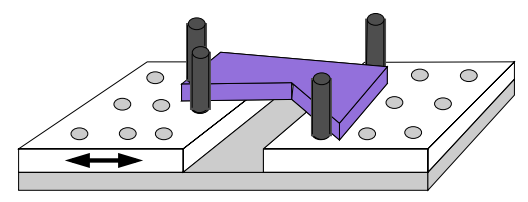

Figure 4: A polygon in form closure on a vise.

their reconfigurability; it is often necessary to fixture an object only for short periods of time after which the same set of fixels can be used to fixture different objects. Another advantage of modular fixtures is their easy assembly and disassembly.

Since research in computing form-closures generally involved point-contacts, it is not surprising that most fixels were designed to achieve point-contact. The simplest fixel is the locator which is a circular object centered at a lattice hole. Since achieving contact with four circles constrained to a grid is in general impossible ([31] shows this even for three circles), it is clear that we need a fixel that takes care of the slack. Such a fixel is called a clamp which has a fixed portion, the clamp body, attached to a movable rod, the clamp plunger, that can translate between certain limits along a grid line. The end of the plunger is the clamp tip which makes contact with the object. A clamp can be configured so that the motion of the plunger is parallel to either one of the axes; it is termed horizontal or vertical accordingly. See Figure 2 for an example of a clamp. An edge-fixel is simply a bar-like object of appropriate dimensions fixed to the lattice offering a straight-edge of support. We assume that an edge-fixel is at least as long as the longest edge in the convex hull of the object. Two edge-contacts are achieved by the use of a so-called angle-fixel, see for example Figure 3. The angle-fixel consist of two edge-fixels that are connected by a joint (an adjustable angle-fixel) or connected to each other with a fixed angle.

Wallack and Canny [29] consider an interesting model of modular fixtures which uses four locators and no clamps; instead, the slack is countered by mounting the part on a split horizontal lattice and allowing the one half to slide horizontally relative to the other. They call such a fixture device a vise. See Figure 4 for an example of this fixture model. 
We will give fixturability results for models using the different fixture elements described above and also give an overview of algorithms to compute all possible form closure configurations on a grid. It is important to generate all solutions rather than just one. The reason is that additional conditions make some solutions preferable over others. By generating all solutions we can compare them and choose the one most suited for the particular problem. We consider both output-sensitive and non output-sensitive algorithms. An algorithm is called output-sensitive if its running time depends on the number of answers (form closure configurations) it computes. A non output-sensitive algorithm has a fixed running time. This is independent from the number of form closure configurations. Usually one prefers output-sensitive algorithms, but it is not always possible to find them.

This overview is structured as follows. We first give a short introduction on the notions of form closure and immobility in Section 2. Then, after introducing some preliminaries in Section 3, we discuss in Section 4 the different types of fixture models that are considered in the rest of the paper. In Section 5 we give an overview of (non-) fixturability results in different models, where we are not restricted to a grid. We also show how to compute one form closure configuration efficiently. Section 6 considers the case of modular fixtures. Finally, we conclude with possible extensions of the algorithms and open problems in Section 7 .

\section{Form Closure and Immobility}

The terminology used in fixturing literature over the past years has not always been consistent. Here we discuss a few different notions on immobility of objects and show how our definition of form closure, which we will use in the rest of this paper, fits in. Several authors have discussed form closure and immobility [3, 11, 24, 26, 27]. Initially Reuleaux [25] used the term force closure to describe immobilization (equilibrium) of an object that requires the application of an externally applied wrench. He defined form closure on a body as an equilibrium that is maintained despite the application of any possible externally applied wrench (force and moment). The method that we use (and that was first described by Reuleaux) is an instantaneous analysis and describes only the constraints on infinitesimal motions. This is equivalent to the notion of $1^{\text {st }}$ order immobility introduced by Rimon and Burdick [26]. $2^{\text {nd }}$ order immobility analysis includes the curvatures of the object and fixel surfaces into the determination of object immobility and therefore leads to other results [10, 26]. Since most of the literature focuses on $1^{\text {st }}$ order immobility, we will define this as form closure. (So whenever we use the term form closure, we mean $1^{\text {st }}$ order immobility.) This notion is a sufficient condition for immobility, but not a necessary one as pointed out by Rimon and Burdick [26]. When taking into account $2^{\text {nd }}$ order immobility, it turns out that three contacts suffice to immobilize almost all two-dimensional objects. The three contact normals, however, must intersect in a single point which is usually hard to accomplish in practice. From a practical point of view it seems better to use $1^{\text {st }}$ order immobility. 

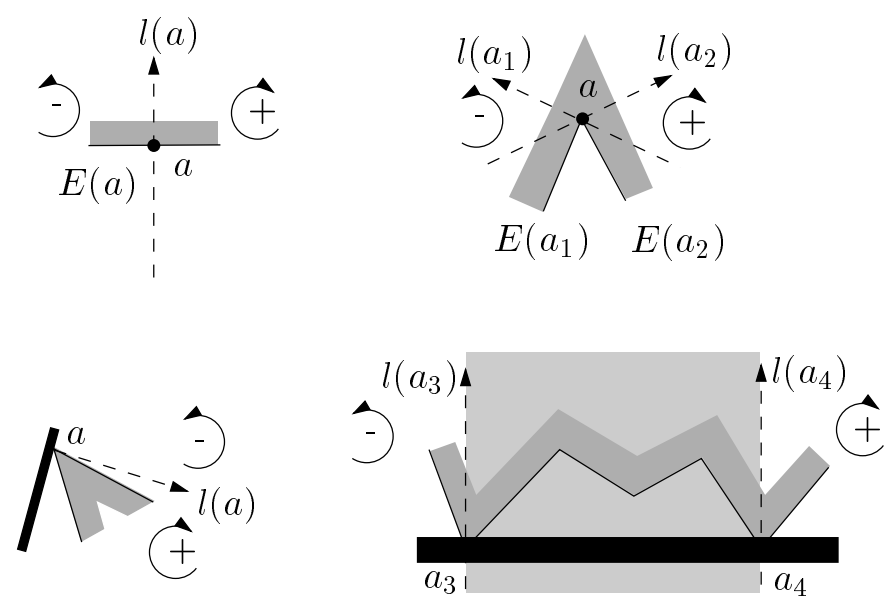

Figure 5: Motions possible under simple contacts.

Furthermore forces that will be applied on the point-contacts can be arbitrary large if we use only three point-contacts (if, for example, we drill a hole at the point where the three normals intersect each other)

\section{Geometric Interpretation}

Recall that a fixture is said to provide form-closure if it precludes all (planar) motion, translations and rotations. Let us start by examining what motions are ruled out by single point- or edge-contacts. Let from now on the object to be fixtured be a polygon $P$ (not necessarily convex). A point-contact is a contact of a point-like fixture element (locator or clamp) with an edge or a vertex of $P$, whereas an edge-contact is a contact of an edge of a fixture element with an edge or one or more vertices of the object $P$. A wrench applied to $P$ will make it translate or rotate. We will consider rotations only, with the understanding that translations in a direction are simply rotations about a point at infinity along the perpendicular direction. In this manner an (infinitesimal) motion of a polygon can be represented by a point in the plane, denoting the center of rotation of this motion; together with the direction of the rotation: clockwise $(+)$ or counter clockwise (-). A point at infinity thus represents an (infinitesimal) translational motion.

Denote the boundary of the input polygon $P$ by $\partial P$; $n$ is the number of edges forming $\partial P$. Let $C H(P)$ denote the convex hull of $P$. Let the polygon edge containing a point $a$ on its boundary be denoted by $E(a)$; the directed line perpendicular to $E(a)$ through $a$ pointing to the interior of $P$ is $l(a)$. We distinguish the following cases (See Figure 5).

\section{Point-contact at interior of an edge}

This is the fundamental contact and the motions allowed by other types of contacts can be deduced by composing those allowed by elementary point-contacts. 
Consider a point-contact at point $a$ in the interior of edge $E(a)$ as shown in Figure 5. The allowed motions are defined by the line $l(a)$. If the object rotates in a clockwise (positive) direction with a point-contact at $a$, its centerof-rotation (COR) will have to lie in the region to the right of (and including) $l(a)$. Furthermore, any point in this closed right-half-plane is a possible COR for a positive rotation. Similarly, the COR's for counter-clockwise or negative rotations lie in the closed half-plane to the left of $l(a)$. These are all and the only constraints imposed by the point-contact at $a$. For future reference, we call these half-plane constraints imposed by $a$.

\section{Point-contact at concave vertex}

This is shown in the upper-right of Figure $5 ; a$ is the concave vertex. Imagine two points $a_{1}, a_{2}$ infinitesimally close to $a$ along the two edges defining it. The motions allowed by $a$ can be determined by intersecting the motions allowed by the point-on-edge pairs $\left(a_{1}, E\left(a_{1}\right)\right)$ and $\left(a_{2}, E\left(a_{2}\right)\right)$ which may be individually analyzed as above.

Consider rotating a line $l$ through $a$ from $l\left(a_{1}\right)$ to $l\left(a_{2}\right)$ in the clockwise direction. The wedge constraints defined by $a$ is the intersection of each of the half-plane constraints obtained along the sweep. Therefore, the motions allowed by $a$ are described by the intersection of two half plane constraints, this can be represented by a wedge. The result is shown in the figure. These will be called wedge constraints defined by $a$.

\section{Edge-contact at a vertex of $C H(P)$}

An edge-fixel can be in contact with a vertex of the convex hull of $P, C H(P)$. Consider the line $l(a)$ perpendicular to the edge-fixel and directed towards the side of the fixel that contains the polygon. All points in the half plane to the left of $l(a)$ can be COR's for counterclockwise rotations. Similarly the points in the right half-plane are COR's for clockwise rotations of $P$. Thus, this type of contact can be described by one half-plane constraint.

\section{Edge-contact at an edge of $C H(P)$}

An edge-fixel can be in contact with the polygon along an edge $e$ the polygon or at two vertices $a_{3}, a_{4}$ (adjacent on the convex hull). The latter case is shown in the Figure 5 ; the former case can be similarly analyzed considering $a_{3}, a_{4}$ to be the end-vertices of $e$. Consider the lines $l\left(a_{3}\right), l\left(a_{4}\right)$ perpendicular to the edge-contact and confine the two half-plane constraints to get the result shown. The closed left half-plane at $l\left(a_{3}\right)$ allows for negative rotations while the right half plane of $l\left(a_{4}\right)$ allows for positive rotations. The open infinite "slab" in the middle, shown shaded in the figure, denoted $\operatorname{slab}\left(a_{3}, a_{4}\right)$, disallows all motions and is a crucial entity in future analysis. The constraint imposed will be called a slab constraint, which is actually a combination of two half-plane constraints.

We do not give constraints for a point-contact at a concave vertex, because in practice, these contacts are not used. These contacts are not stable and will lead to deformation of the part if the forces become too large.

If we want to know if an object is in form closure, we have to construct 


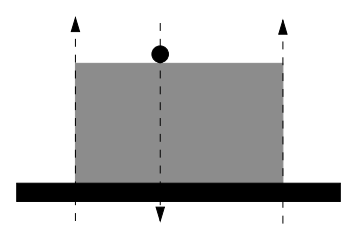

Figure 6: A rectangle that is not fixed with one point-contact and one edgecontact.

the regions associated with the contacts that are imposed and confine them. If there is no region that allows for positive (or negative) rotations for every constraint, we have a form closure configuration, otherwise (infinitely small) motions are possible. The method of analysing possible motions in this geometrical (graphical) way was introduced by Reuleaux [25] in the case of point-contacts. It should be noted that two (or more) parallel contact normals intersect in a point at infinity. See for an example Figure 6, in this case all positive and negative rotations seem to be excluded, but the intersection point at infinity still gives a possible center of rotation. Since this point is at infinity, a rotation means translation, thus the rectangle can still be translated to the left or to the right.

\section{Fixture Models}

In this paper we will consider four different ways of fixturing objects: with four point-contacts, with one edge-contact and two point-contacts and two edgecontacts and one point-contact (of which two perpendicular edge-contacts and one point-contact is a special case). All these models are minimal in the sense that for most polygons we need all the fixels in the model to achieve a form closure configuration. We can make a comparison of the different fixture models using a number of criteria. The main criteria that we discuss here are classes of fixturable objects (which we will call the strength of the model, the model being stronger if more objects can be fixtured), loading the part, accessability, efficiency of computation of fixtures and stability of the fixtures. We also compare the number of contacts that are made in the model in terms of half-plane constraints. In the rest of the paper we will assume that whenever we use one or more edge-contacts, one of these edge-contacts will be an edge-contact at an edge of $C H(P)$.

\section{Four point-contacts}

This is the basic model in which all polygons can be fixtured. In the following we will discuss why we also look at other fixture models that seem to be less strong, but do have advantages over the four-point model. A model with four point-contacts is preferred if we need to have a large accessability of the part, since edge-contacts will constrain accessability of $P$ more than point-contacts. The maximum number of half plane constraints that we can get in this model 
is eight, if we place all four contacts at concave vertices of the polygon.

\section{One edge and two point-contacts}

An edge-fixel placed against an edge of $C H(P)$ imposes two half-plane constraints on $P$. The two point contacts can result in a maximum of four halfplane constraints if we place them at concave vertices. The model is not as strong as a model with four point-contacts, because we cannot fixture some parts that have parallel edges, as will be shown below. The reason for this is that the two point-contacts as imposed by the edge are colinear. The advantage of the system however is that the computation of all possible fixtures in a modular model can be done more efficiently, because $P$ can only be in a fixed number of different orientations, which is not the case when we only have point-contacts. Intuitively, the stability of a fixture with an edge-fixel is better when forces are applied to it, because the force will be distributed along the edge and not only act on two points. We also believe that it is easier to load an object into the fixture even when the initial orientation of $P$ is uncertain. If we first load the polygon against the edge-fixel, we already have fixed the orientation of $P$ and can then slide it against the locator and finally apply the clamp.

\section{Two perpendicular edge-contacts and one point-contact}

In this case, again we can not fixture some parts that have parallel edges. Although the model can impose 5 half-plane constraints (or 6 in the case that two perpendicular edges of $P$ are placed against the edge-fixels), we can not fixture every polygon because there is a dependancy between the three half-plane constraints that are imposed by the edge-fixels (the directions of the contact normals and the contact point with the second edge, when we have fixed the edge against the first edge depend on each other). Once we have determined the edge of $C H(P)$ to place against the horizontal edge-fixel, we have fixed three of the half-plane constraints already. The computation of all possible fixtures is fairly easy. The number of orientations for $P$ is the same as in the model with one edge-contact and two point-contacts. In addition, for each orientation there is only the position for the point-contact to consider. Loading the part seems to be quite easy in the model and intuitively the stability of the fixtures is good.

\section{Two edge-contacts and one point-contact}

With this model we have more degrees of freedom than in the case of two perpendicular edge-fixels, since we can vary the angle between the two edge-fixels. As a result, it turns out that we can fixture all polygons. In the model we can have maximal 6 half-plane constraints, two for each edge-contact and two for the point-contact (if it is placed at a concave vertex). In addition however, these are not always the placements that will give us a form closure configuration (e.g. a rectangle). But, if this is not the case, we can find a form closure configuration with a different placement of the part (if we assume that there is no grid). A disadvantage of the model is that the edges should be positionable in any orientation, which is not always achievable in practise.

So, in conclusion we can say that the models with four point-contacts and with 


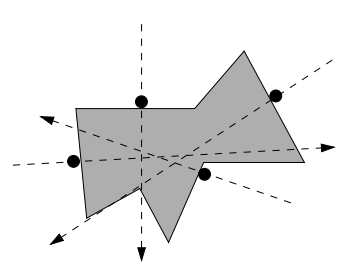

Figure 7: A polygon that is fixtured with four point-contacts.

two edges and one point-contact are the strongest but they have the disadvantages that form closure configurations are harder to compute, they are more difficult to load and less stable.

\section{$5 \quad$ Fixturability without a Grid}

In this chapter we examine what kind of objects are fixturable with each of the toolkits mentioned in the previous section. We do not yet take modularity constraints into account. For every model we show what is known about fixturability of classes of objects and algorithms to compute one form closure configuration. We will frequently use a property of the contact points of the maximal inscribed circle of $P$ with the boundary of $P$ as is stated in the following lemma. The lemma follows from Markenscoff et al. [19].

Lemma 1 [19] Let $P$ be a polygon without pairs of parallel edges. Let $M I C(P)$ be any maximal inscribed circle of $P$ and $c$ the center of $M I C(P)$. Then the three vectors $\overrightarrow{c a_{i}}, a_{i} \in(M I C(P) \cap \partial P)$, positively span $\mathbf{R}^{2}$.

The time to compute the maximal inscribed circle of a polygon $P(M I C(P))$ is $O(n)$ both for convex and non-convex polygons [7]. This can be done by computation of the medial axis of $P$, the center of the $M I C(P)$ is a vertex of the medial axis of $P$. We also use the following lemma that can easily be proved.

Lemma 2 Given a set $V=v_{1}, v_{2}, v_{3}$ of three vectors that positively span $\mathbf{R}^{2}$. For every fourth vector $v_{4}$ in $\mathbf{R}^{2}$, we can define a new set of vectors consisting of $v_{4}$ and two vectors from $V$, such that this new set positively spans $\mathbf{R}^{2}$.

\subsection{Four Point-contacts}

Since the graphical method of Reuleaux from Section 3 is not always the easiest test for automatically verifying for form closure, other methods for testing form closure with four point contacts are commonly used. A point-contactvector on an object in two dimensions can be described by three variables; the position of the contact and its torque. Four point contacts provide form closure if and only if the four associated point-contact-vectors positively span $\mathbf{R}^{3}$. This can be verified using matrix computations [29] or force-sphere analysis [6]. Markenscoff et al. [19] proved the following Theorem. 


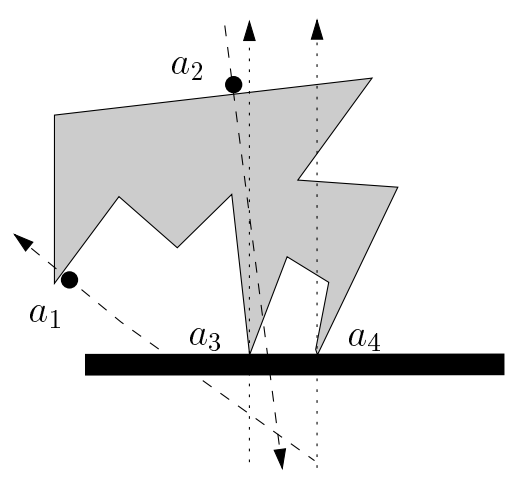

Figure 8: A polygon fixtured with one edge-fixel and two point-fixels.

Theorem 3 [19] For any object $O$ that is not a circle there is a set of four point contacts (possibly at a concave vertex) that provides form closure.

The proof uses the properties of the maximum inscribed circle $(M I C(P))$ as stated in Lemma 1. The proof utilizes point contacts that are either on or close to the points of contact of a maximum inscribed circle of the object. It distinguishes two cases.

First there is the case where there are three contact points of $M I C(P)$ with $P$. In this case one of these points is replaced by two points that are close to each other and on either side of the contact point of $M I C(P)$ and $P$. It is then shown that the point-contact-vectors at these four points positively span $\mathbf{R}^{3}$. The second case is the case where (some of the) $M I C(P)$ 's touch only two points of $P$. This only happens when the $M I C(P)$ touches two parallel edges of $P$. In that case the $M I C$ can be slided along these two edges, while its size remains the same. Thus we do not have a unique $M I C$. The intersection points of $M I C(P)$ and $P$ are antipodal. If we assume that there is a third point on some $M I C(P)$ that touches $P$, we can always find a fourth point that will give us a form closure configuration. If there is no such a third contact point all the $M I C(P)$ 's touch the boundary only in two points. In this case we can move the $M I C(P)$ to the left and the right in order to obtain two couples of forces that will give a so-called form closure by couples.

The proof is constructive in the sense that it does not only show the fixturability of objects in the four-point model, but it also indicates how to compute a form closure configuration for every two-dimensional object, by computing its $M I C$, leading to an algorithm with a running time of $O(n)$.

\subsection{One Edge-fixel and Two Point-fixels}

The problem of immobilizing a polygon with one edge-contact (where the edge-fixel is placed against a convex-hull-edge of $P$ ) and two point-contacts was analysed by Overmars et al. [23]. In Section 3 we have described what motions are constrained by an edge-edge contact and a point-edge contact. To constrain all motions of the object the set of allowable centers of rotation must be empty. The following lemma gives a more useful condition for form closure 


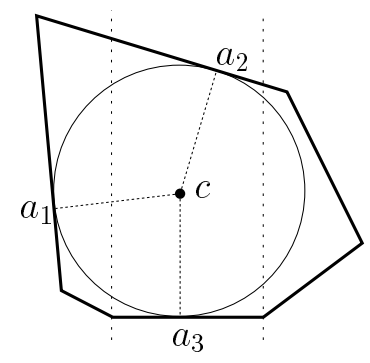

Figure 9: The tangent points of the largest inscribed circle of $P$ with $P$ give us the edge- and point-contacts for form-closure.

in this model. Let $a_{1}$ and $a_{2}$ be two points on an edge of $P$, and $a_{3}$ and $a_{4}$ two adjacent vertices on $C H(P)$.

Lemma 4 [23] An object $P$ is in form-closure with point-contacts $a_{1}, a_{2}$ and edge-contact $\left(a_{3}, a_{4}\right)$ if and only if

1. the three vectors along $l\left(a_{1}\right), l\left(a_{2}\right), l\left(a_{3}\right)$ positively span $\mathbf{R}^{2}$, and

2. the intersection point of $l\left(a_{1}\right)$ and $l\left(a_{2}\right)$ lies in the interior of slab $\left(a_{3}, a_{4}\right)$.

See Figure 8 for an example of an object that is in form closure with one edgecontact and two point-contacts.

Overmars et al. showed that if no edges of $P$ are parallel then a form closure configuration always exists [23]. Their proof goes as follows. If we have the maximal inscribed circle of a convex polygon without parallel edges, there are always (at least) three intersection points $\left(a_{1}, a_{2}, a_{3}\right)$ of $P$ and $M I C(P)$, that lie in the interior of edges of $P$ and whose contact normals positively span $\mathbf{R}^{2}$ (Lemma 1). If we place one of these edges against the edge-fixel, we obtain form closure by placing the two point-contacts at the other two intersection points of $P$ and $M I C(P)$. This is true because the contact normals to these two points will intersect at the center $(c)$ of $M I C(P)$ and the slab defined by the edgecontact has $c$ strictly in its interior. From Lemma 4 we can now conclude that this is a form closure configuration. See for an example Figure 9.

A similar proof can be given if $P$ is not convex. If at least one of the edges of $C H(P)$ is on the $M I C(P)$ we can use the same form closure construction as for a convex polygon, since the edge-fixel can then be placed against this edge of $C H(P)$. If none of the intersection points of $P$ and $M I C(P)$ is on a convex hull edge of $P$ we cannot place any of these edges against the edge-fixel. Instead, we grow the circle until we touch an edge of $C H(P)$. We can then place this edge against the edge-fixel and choose two points of $\left(a_{1}, a_{2}, a_{3}\right)$ whose contact normals will span $\mathbf{R}^{2}$ together with the direction of the edge-contact normal (corresponding to the edge-contact) using Lemma 2. See for example Figure 10. Thus we obtain the following Theorem. 


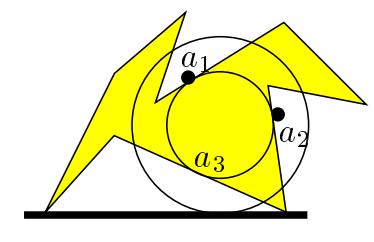

Figure 10: Growing the maximal inscribed circle of a non-convex polygon, until it hits an edge of $C H(P)$ gives a form closure configuration.

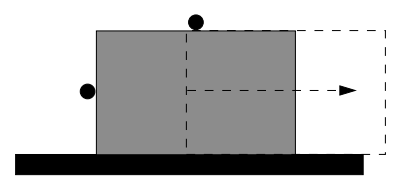

Figure 11: A rectangle can not be fixtured in the model with one edge-contact and two point-contacts. We can always still translate it in horizontal or vertical direction.

Theorem 5 [23] Let $P$ be an arbitrary polygon. If no edge of $P$ is parallel to one of the edges of $C H(P)$ then $P$ can be held in form closure with one edgeand two point-contacts.

However, it is not true that all polygons that do have parallel edges cannot be fixtured. We can even show that rectilinear polygons of which the convexhull is not a rectangle are fixturable with an edge-fixel and two point-contacts.

The proof is again constructive, leading to an $O(n)$ algorithm for computing one form closure configuration.

Not all polygons can be fixtured in this model, for example a rectangle and some trapezoids cannot be fixtured as shown in Figures 11 and 12. The rectangle can always be translated in horizontal or vertical direction if we place the point-contacts at interiors of edges of $P$. The trapezoid with two parallel edges, $a$ and $c$ can not be fixtured. Consider all possibilities to place one edge of the trapezoid against the edge-fixel, then it can be seen that none of the correctly oriented contact normals will intersect in the slab defined by the edge-contact. Thus, according to Lemma 4 a form closure configuration does not exist.

\subsection{One Angle-fixel and a Point-fixel}

In a fixture model with one angle-fixel and a point-fixel, there are two possible sets of contacts. The angle-fixel can sometimes be placed such that both edges of the fixel make contact with an edge of the convex-hull of $P$. In this case the intersection between the two slabs defined by the edge-contacts is a parallelogram, $s$. For an example see Figure 13. If we want to obtain a formclosure configuration the contact normal to the point-contact should intersect 


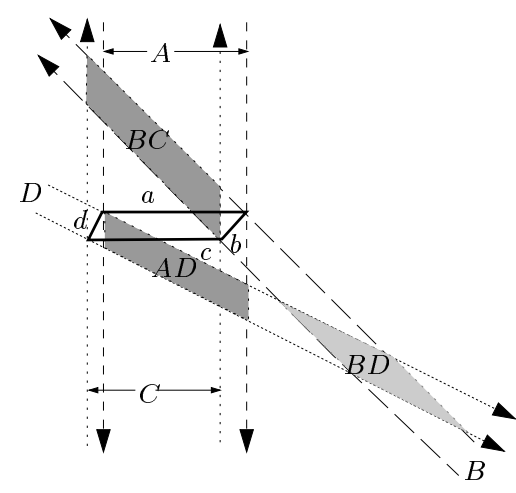

Figure 12: Trapezoid $a b c d$ can not be fixtured in a model with one edge-fixel and two point-fixels, since the intersection of two slabs is never intersected by a third slab that is correctly oriented.

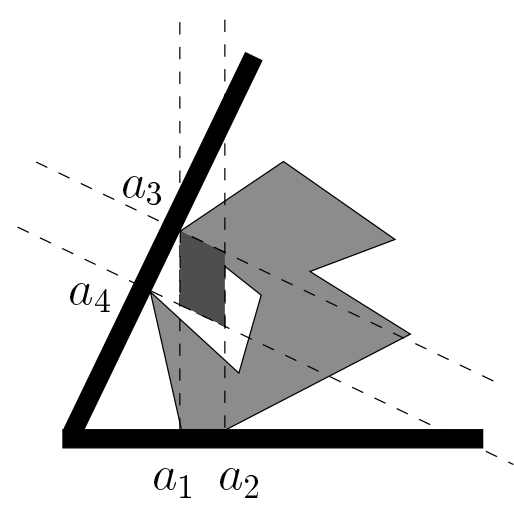

Figure 13: A polygon in contact with an angle-fixel, where the two contacts are both edge-contacts.

the parallelogram and be correctly oriented. Let $a_{1}$ be the the leftmost vertex contacting the first edge-contact and $a_{2}$ the rightmost vertex contacting this edge. Similarly $a_{3}$ is the leftmost vertex contacting the second edge and $a_{4}$ the rightmost contact vertex.

Lemma 6 An object $P$ is in form-closure with edge-contacts $\left(a_{1}, a_{2}\right)$ and $\left(a_{3}, a_{4}\right)$ and point-contact $a_{5}$ if and only if

1. The three vectors along $l\left(a_{1}\right), l\left(a_{3}\right), l\left(a_{5}\right)$ positively span $\mathbf{R}^{2}$, and

2. $l\left(a_{5}\right)$ should intersect the interior of $\operatorname{slab}\left(a_{1}, a_{2}\right) \cap \operatorname{slab}\left(a_{3}, a_{4}\right)$.

In the other case one edge-fixel can be placed against an edge of $C H(P)$ (this contact will define a slab-constraint) and the other edge will contact $P$ at a vertex of the convex hull (imposing an edge-vertex constraint as described in 


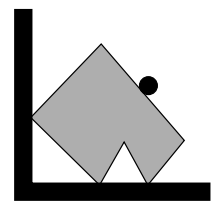

LRA-fixel

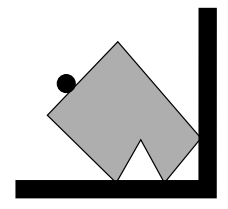

RRA-fixel

Figure 14: A polygon in form closure with a LRA-fixel and a point contact and with a RRA-fixel and a point-contact.

Chapter 3). If we intersect these two constraints (i.e. a slab and a line), we obtain a segment, $s$. In fact this is a special case of Lemma 6 , where $a_{3}$ and $a_{4}$ coincide, Thus reducing the parallelogram to a segment. We will consider two different cases. One in which the two edge-fixels make a right angle and one in which the angle can be chosen arbitrarily.

\subsubsection{Right Angle-fixel and a Point}

In this section we investigate fixturing an object with a right-angle-fixel and a point-fixel. A right-angle fixel offers two edge-contacts, perpendicular to each other. See Figure 14 for an example. We assume one of the edges to be horizontal. We will consider two possible orientations of the fixel that will give different form closure configurations. One way of placing the angle-fixel is such that the second (vertical) edge will be to the left of the object (LRA-fixel) and the other placement where the second edge is to the right of $P$ (RRA-fixel). See Figure 14 for the two placements of the RA-fixel. An object is called fixturable in the model if it can be fixtured with at least one of the orientations of the RA-fixel. A rectangle can not be fixtured, since no triple of contact normals can positively span $\mathbf{R}^{2}$. Currently, we have no other examples of polygons that cannot be fixtured in this model.

We will now show that any convex polygon without parallel edges can be fixtured. For non-convex polygons this is still an open problem. The pair of boundary features (edges, vertex) of a convex polygon $P$ intersected by the two closest parallel lines of support of $P$ satisfies a property that turns out to be useful the proof of Theorem 8. A supporting line of $P$ either intersects $P$ in a vertex or along an edge. A pair of boundary features intersected by two parallel supporting lines is referred to as an antipodal pair. Note that in the case that the polygon $P$ has no parallel edges, an antipodal pair consists of either two vertices or one edge and one vertex. The distance between the two closest parallel lines of support is referred to as the width of the polygon. The antipodal pair that determines the width of $P$, i.e., the pair of features that are intersected by the two closest parallel supporting lines, consists of an edge $e_{w}$ and a vertex $v_{w}[15]$. The pair $\left(e_{w}, v_{w}\right)$ can be computed in time linear in the number of polygon vertices, Lemma 7 gives an interesting property of the antipodal pair determining the width. 
Lemma 7 Let $P$ be a convex polygon without parallel edges and let $v_{w}$ be the vertex and $e_{w}$ be the edge intersected by the two closest parallel supporting lines of $P$. Then the line through $v_{w}$ and perpendicular to $e_{w}$ intersects the interior $e_{w}$.

Proof: The lemma is true if and only if $v_{w}$ lies in the open slab $\operatorname{sl}\left(e_{w}\right)$ of all lines that perpendicularly intersect the edge $e_{w}$. Assume without loss of generality that the edge $e_{w}$ and the two closest parallel supporting lines of $P$ are horizontal, as in Figure 15. As a result, the slab $s l\left(e_{w}\right)$ is vertical. Assume, for a contradiction, that $v_{w}$ does not lie in the slab $\operatorname{sl}\left(e_{w}\right)$. Let us assume that $v_{w}$ lies to the right of the slab. Let $c$ be the right endpoint of the edge $e_{w}$. Recall that the (horizontal) lines of support at $v_{w}$ and $e_{w}$ are the two closest parallel lines of support of the polygon. By the assumption that no two edges

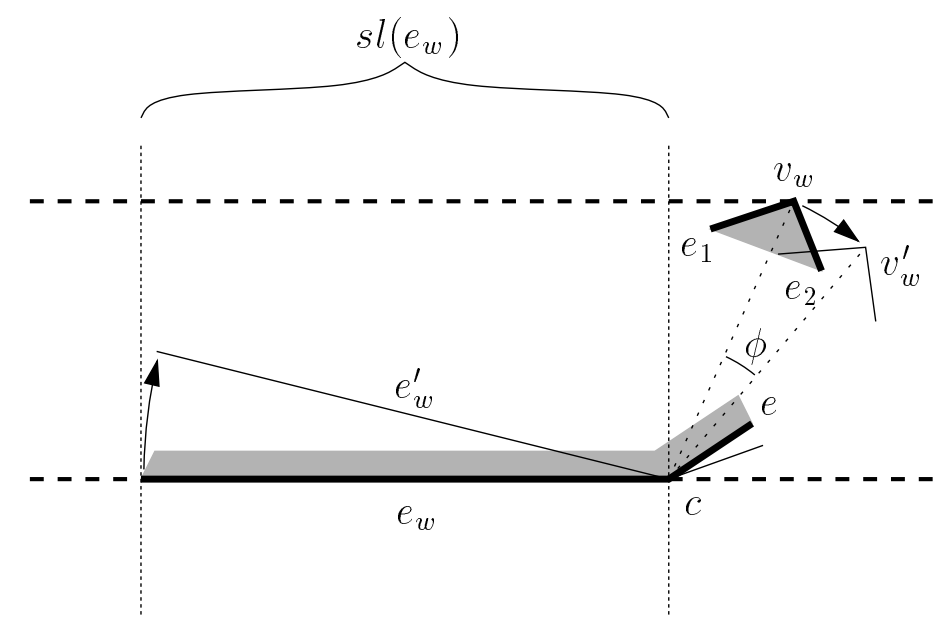

Figure 15: When $v_{w}$ lies to the right of the slab $\operatorname{sl}\left(e_{w}\right)$ of lines that perpendicularly intersect $e_{w}$, a small clockwise rotation about $c$ causes the polygon to loose contact with the upper horizontal line.

of the polygon are parallel, neither the edge $e$ incident to $c$ nor the edge $e_{1}$ incident to $v_{w}$ can be horizontal. The convexity of the polygon implies that it is entirely contained in the wedge bounded by the supporting half-lines of $e_{1}$ and $e_{2}$ emanating from $v_{w}$ and in the wedge bounded by the supporting half-lines of $e_{w}$ and $e$ emanating from $c$. Let $\phi$ be some arbitrary angle smaller than the angle between the edge $e$ and the lower horizontal line and the angle between the edge $e_{1}$ and the upper horizontal line. A clockwise rotation of $\phi$ about $c$ causes the wedge at $v_{w}$ to fall strictly below the upper horizontal line, whereas the wedge at $c$ remains above the lower horizontal line. As a result, the distance between the two horizontal lines of support can be decreased by lowering the upper line, contradicting the fact that the initial horizontal lines are the closest parallel lines of support. Hence, the vertex $v_{w}$ cannot lie to the right of the slab $s l\left(e_{w}\right)$. Similar arguments apply if $v_{w}$ lies to the left of the slab $s l\left(e_{w}\right)$, using a counterclockwise rotation about the left endpoint of $e_{w}$. 


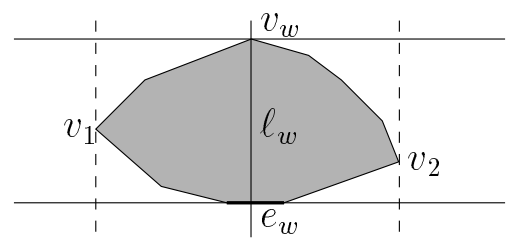

Figure 16: The line $\ell_{w}$ through $v_{w}$ perpendicularly intersects $e_{w}$. The vertical lines of support intersect the polygon in the vertices $v_{1}$ and $v_{2}$.

Thus, the vertex $v_{w}$ must lie inside the slab $\operatorname{sl}\left(e_{w}\right)$ of lines that perpendicularly intersect $e_{w}$. Hence, $v_{w}$ must be on a line that perpendicularly intersects $e_{w}$.

Informally, Lemma 7 tells us that we can put any convex polygon without parallel edges into an orientation where one of its edges is horizontal and the highest (top) vertex lies above that horizontal edge. This will be the orientation in which we will fixture the object.

Theorem 8 Any convex polygon without parallel edges can be immobilized by a right angle fixel and a single point contact.

Proof: Let $e_{w}$ be the edge and $v_{w}$ be the vertex intersected by the two closest parallel supporting lines of the polygon. Let $\ell_{w}$ be the line through $v_{w}$ and perpendicular to the edge $e_{w}$, which, by Lemma 7 , intersects the interior of $e_{w}$. We assume, without loss of generality, that the edge $e_{w}$ and the two closest parallel supporting lines are horizontal. The line $\ell_{w}$ is vertical.

Consider the two lines of support that are perpendicular to the two closest parallel supporting lines (see Figure 16).

Each of these lines intersects the polygon boundary in a vertex; in the accidental case that the line intersects the polygon along an edge, simply take its top endpoint to be the vertex of intersection. Note that the two resulting vertices $v_{1}$ and $v_{2}$ are the candidate contact points with the second fixel bar if one fixel bar is placed along $e_{w}$. Choose $v_{v}$ to be the highest (in vertical direction) of the two vertices $v_{1}$ and $v_{2}$. We place the angle fixel in simultaneous contact with the full edge $e_{w}$ and the vertex $v_{v}$. Let $\ell_{v}$ be the horizontal line through $v_{v}$, and let $q$ be its intersection point with $\ell_{w}$. The point $q$ lies strictly below $v_{w}$. Note that the contact normal at $v_{v}$ is directed along $\ell_{v}$ and pointing towards the interior of the polygon. The contact normals at $e_{w}$ point upward.

It remains to show that there exists a point $p$ on the polygon boundary such that the right angle fixel placed against $e_{w}$ and $v_{v}$ and a point contact at $p$ immobilize the polygon. More specifically, we show that there exists a point $p$ such that (i) the contact normal at $p$ positively spans the plane with the contact normals at $v_{v}$ and $e_{w}$ and (ii) the supporting line $l(p)$ of the contact normal at $p$ intersects the supporting line $\ell_{v}$ of the contact normal at $v_{v}$ in $q$, which is contained in the slab induced by the edge contact at $e_{w}$. By Lemma 6 , this will imply form closure. 


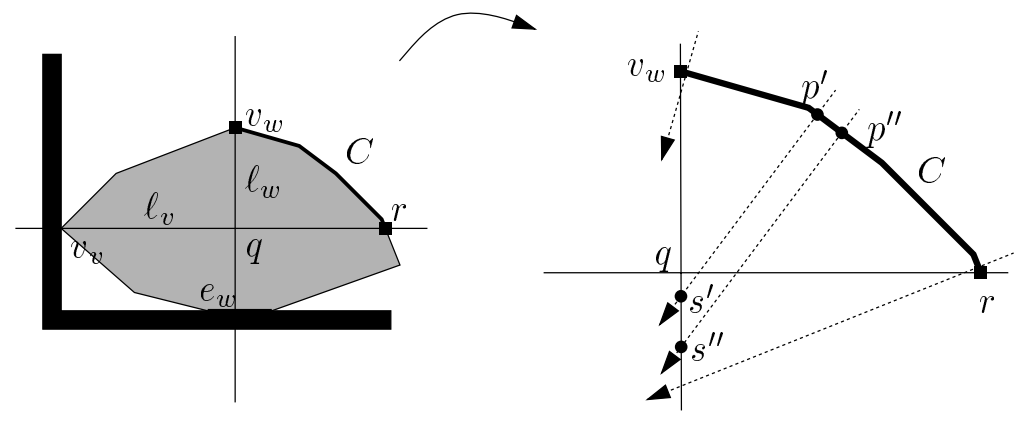

Figure 17: The angle fixel is placed in simultaneous contact with $e_{w}$ and $v_{v}$. A point contact $p$ can be placed on the convex polygonal chain $C$ such that $p$ and the angle fixel hold the polygon in form closure. The closer view of $C$ on the right shows that if $p$ moves from $p^{\prime}$ to $p^{\prime \prime}$ along an edge of $C$, the intersection $s$ of $l(p)$ and $\ell_{w}$ moves from $s^{\prime}$ to $s^{\prime \prime}$. Given the initial and final positions of $s$ on $\ell_{w}$, the point $s$ must eventually pass through $q$.

Now assume that $v_{v}$ is the vertex intersected by the left vertical supporting line. The contact normal at $v_{v}$ points to the right. Figure 17 shows the situation. We claim that there is a point $p$ satisfying (i) and (ii) on the convex polygonal chain $C$ to the right of $\ell_{w}$ and above $\ell_{v}$. The chain is strictly decreasing and contains no vertical edges by the observation that the vertex of intersection with the right vertical supporting line of the polygon lies below $\ell_{v}$. As a result, the contact normal at any point $p \in C$ satisfies condition (i). Let $r$ be the endpoint of $C$ on $\ell_{v}$.

Let us consider the motion along $\ell_{w}$ of the intersection point $s$ of $l(p)$ and $\ell_{w}$ when $p$ moves from $v_{w}$ to $r$ (see Figure 17). By placing $p$ sufficiently close to $v_{w}$ on $C$, we can put the intersection point $s$ arbitrarily close to $v_{w}$, and, hence, above $q$. By placing $p$ at $r$ or sufficiently close to $r$ on $C$ if $r$ is a vertex - we can put $s$ below $q$ by the fact that $r$ is on a non-vertical edge. If $p$ moves towards $r$ along some edge of $C$ then the intersection point $s$ moves downward along $\ell_{w}$ (see Figure 17 where $s$ moves from $s^{\prime}$ to $s^{\prime \prime}$ as $p$ moves from $p^{\prime}$ to $p^{\prime \prime}$ ); if $p$ passes a vertex then $s$ jumps back up. As a consequence, the point $s$ will pass through $q$ as $p$ moves along one of the edges of $C$ on its way from $v_{w}$ to $r$. Hence, there exists a point $p$ that satisfies conditions (i) and (ii). As a result, a point contact at $p$ establishes form closure for the polygon placed against the angle fixel. Similar arguments apply when $v_{v}$ is the vertex intersected by the right vertical supporting line.

This fixturability-proof can be generalized to angle-fixels with an arbitrary fixed angle, we will not give the proof here, but only state the corresponding theorem.

Theorem 9 Any convex polygon without parallel edges can be immobilized by a fixed angle fixel and a single point contact.

Since computation of the width of $P$ takes $O(n)[15]$ and testing the edges 


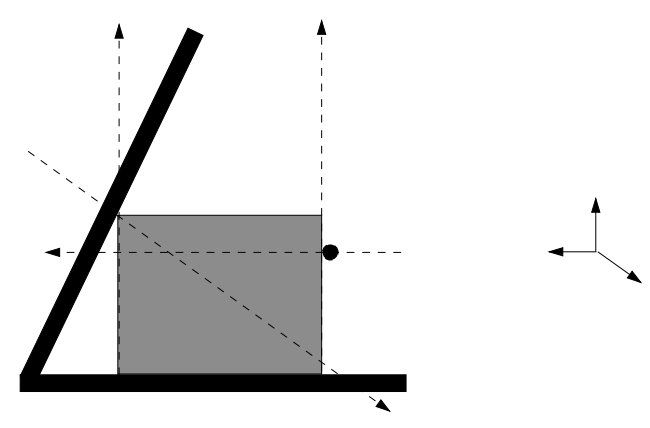

Figure 18: A rectangle fixtured by an angle-fixel and a point-fixel.

on the chain $C$ takes $O(1)$ per edge, a form closure configuration can be computed in linear time.

\subsubsection{Adjustable Angle-fixel and a Point-fixel}

In this section we consider fixtures with an adjustable angle fixel as shown in Figure 18 and a point contact. The angle-fixel is horizontally fixed, but the second edge can be adjusted by rotation about the joint that connects the two edges of the fixel. The second edge can be either to the left or to the right of the horizontally fixed edge. The most stable fixtures can be obtained if two edges of $C H(P)$ are in contact with the edge-fixels. We can prove that there is such a fixture for all polygons without parallel edges. In the case where we do have parallel edges (e.g. a rectangle) we may not be able to fixture $P$ in this manner, but one of the contacts with the angle-fixel should be a point-edge-contact. We will prove fixturability of all polygons using these types of contacts. We use the following lemma.

Lemma 10 Given a polygon $P$ and a point $c$ in the interior of $P$. If we grow a circle with center $c$, the first point that will be hit on the boundary of $P$ is a point on an edge of $P$, tangent to the circle or a concave vertex of $P$. Similarly, if we grow a circle with center $c$ in the exterior of $P$, we will first hit a convex vertex of $P$ or an edge of $P$.

We first deal with the case in which no two edges of the convex hull of $P$ are parallel.

Theorem 11 Let $P$ be an arbitrary polygon. If no edges of $C H(P)$ are parallel then $P$ can be held in form closure with an adjustable angle fixel and a pointcontact.

Proof: Let $M I C(C H(P))$ be the maximum inscribed circle of $C H(P)$. The center of $M I C(C H(P))$ is called $c$. Since $P$ has no parallel edges, $M I C(C H(P))$ will touch $C H(P)$ in at least three points: $a_{1}, a_{2}, a_{3}$. Let $e_{i}$ be the convex hull edge containing $a_{i}$ and let $d_{i}$ be the direction of the contact normal at $a_{i}$ 


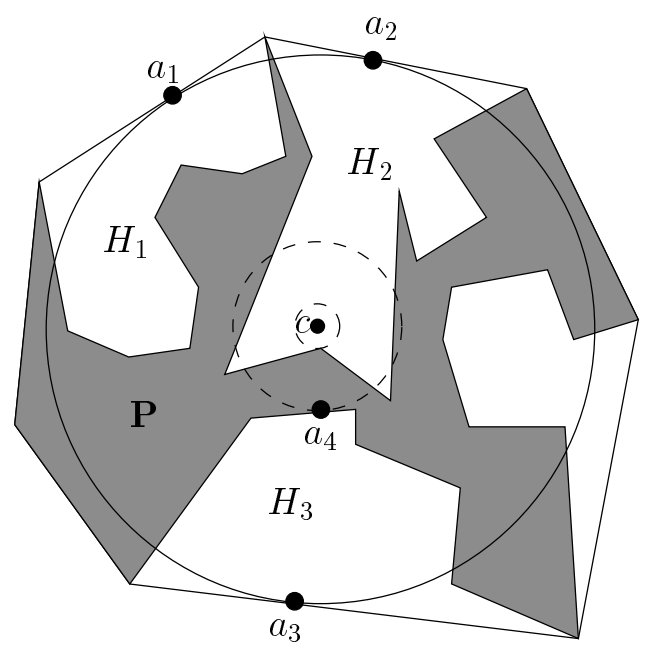

Figure 19: Computation of the contact point if $a_{1}, a_{2}$ and $a_{3}$ are not on the boundary of $P$.

$(1 \leq i \leq 3)$ The vectors $\left(d_{1}, d_{2}, d_{3}\right)$ positively span $\mathbf{R}^{2}$.

If (at least) one of the edges $e_{1}, e_{2}$ or $e_{3}$ is an edge of $P$ itself, say $e_{1}$, we can place the point contact at $a_{1}$ and the edge contacts at $e_{2}$ and $e_{3}$, it follows from Lemma 6 that we have a form closure configuration now. If none of the edges $e_{1}, e_{2}$ or $e_{3}$ is an edge of $P$, we have to find another contact point. We distinguish two cases.

Case 1. Point $c$ is in the interior of $P$.

We grow a circle centered at $c$ inside $P$, until we touch $P$ either at a concave vertex or on an edge of $P$ (Lemma 10). Let the contact point be $a_{4}$. The direction of the corresponding contact normal, $d_{4}$ will span $\mathbf{R}^{2}$ together with two vectors of $V$ according to Lemma 2. Suppose these vectors are $d_{1}$ and $d_{2}$. Now we can position $P$ such that $e_{1}$ and $e_{2}$ are the edge contacts and the point contact is placed at $a_{4}$. Since the contact normals intersect in $c$, this is a form closure configuration.

Case 2. Point $c$ is in the exterior of $P$.

Since none of the distinct edges $e_{1}, e_{2}$ or $e_{3}$ belongs to $P$, each of the points $a_{1}, a_{2}$ and $a_{3}$ lie in cavities $H_{1}, H_{2}$ and $H_{3}$ of $P$ (A cavity is a non-empty polygon bounded by the polygon edges connecting two adjacent convex hull vertices and the convex hull edge connecting these two vertices). Assume that $H_{3}$ is one of the cavities that does not contain $c$ (as $c$ can lie in only one cavity). Thus, by growing a circle from $c$, we will hit a convex vertex of $H_{3}$ (which is a concave vertex of $P$ ) or an edge of $H_{3}$ that is also an edge of $P$. The place where we hit $H_{3}, a_{4}$, is the position of the contact point. From $d_{1}, d_{2}$ or $d_{3}$ we can now select the two vectors that will positively span $\mathbf{R}^{2}$ together with the contact normal $d_{4}$ at $a_{4}$. The corresponding edges of $C H(P)$ will provide form closure by making them the edge contacts. 
Figure 18 suggests that polygons with parallel edges are fixturable in a model with an adjustable angle-fixel and a point-contact. We will prove this for convex polygons. In this case the fixel-edges cannot both be placed against convex-hull edges of $P$, because this can not always give us a form closure configuration as the example with the rectangle shows.

Theorem 12 For every convex polygon $P$ a form closure configuration exists in the model with one adjustable angle-fixel and a point-contact.

Proof: We only give a sketch of the proof.

From the proof of Theorem 11 we can deduce that the problem with parallel edges arises when the maximal inscribed circle touches two parallel edges. Let us call these edges $e_{1}$ and $e_{2}$. The contact points are $a_{1}$ and $a_{2}$ resp. Observe that not all four angles of $e_{1}$ and $e_{2}$ with their adjacent edges can be acute angles (viewed from the interior of $P$ ). Otherwise $P$ would not have been a convex polygon. Choose one of the edges $\left(e_{1}, e_{2}\right)$ that has at least one obtuse angle with an adjacent edge. Without loss of generality suppose this edge is $e_{2}$. We can now show that there is always a form closure configuration such that $e_{1}$ is placed against the horizontal edge.

Let $l$ be the line through $a_{1}$ and $a_{2}$, thus $l$ also contains the center of $M I C(P), c$. The line $l$ is a vertical line, since we have placed $e_{1}$ horizontally, and passes through the center $c$ of $M I C(P)$. Our goal is now to find two contact normals that are correctly oriented (they have to positively span $\mathbf{R}^{2}$ together with the upward pointing contact normal at $e_{1}$ ) and have their intersection point on $l$. Since $l$ is contained in the slab defined by $e_{1}$, we then have a form closure configuration. More precisely, we want to find one contact normal with direction in $I_{1}=\left(-\frac{1}{2} \pi, 0\right)$ and one with orientation in $I_{2}=\left[\pi, 1 \frac{1}{2} \pi\right)$, that intersect on $l$. Since $P$ is convex, the contact normals associated with interval $I_{1}$ will originate from contacts to the left of $l$ and those associated with $I_{2}$ from the right of $l$. We now proceed by distinguishing two cases:

Case 1. One of the edges adjacent to $e_{2}$ forms an acute angle with $e_{2}$, the other one an obtuse angle. An angle of $\frac{1}{2} \pi$ will be considered an obtuse angle. Without loss of generality we assume the acute angle to be to the left of $e_{2}$ and the obtuse angle to the right. For an example, see Figure 20.

In this case the edge-fixel will be placed at the vertex of the acute angle adjacent to $e_{2}$. Let this vertex be $v_{1}$. By adjusting the angle of the edge-fixel from 0 to $\frac{1}{2} \pi$, we will rotate the edge of the fixel around $v_{1}$. Since the angle is acute and $P$ convex, we can always do this without collisions with edges of $P$. The orientations of the contact normals will during the rotation increase from $-\frac{1}{2} \pi$ to 0 . At the same time the intersection point of the contact normal at $v_{1}$ with $l$ will travel (back and forth) along $l$ from $y=-\infty$ to $y=y_{a_{2}}$, where $y_{a_{2}}$ is the $y$-coordinate of $a_{2}$. In other words, for each point on $l$ below $a_{2}$, there exists an orientation of the fixel edge such that the contact normal at $v_{1}$ passes through that point and has an orientation in the range $I_{1}$.

The point contact $a_{c}$ will be placed somewhere at the interior of the edge adjacent to $e_{2}$ on the right side. Since the angle formed by this edge with $e_{2}$ is obtuse, we know that the contact normal at $e_{2}$ will be in the interval $I_{2}$. 


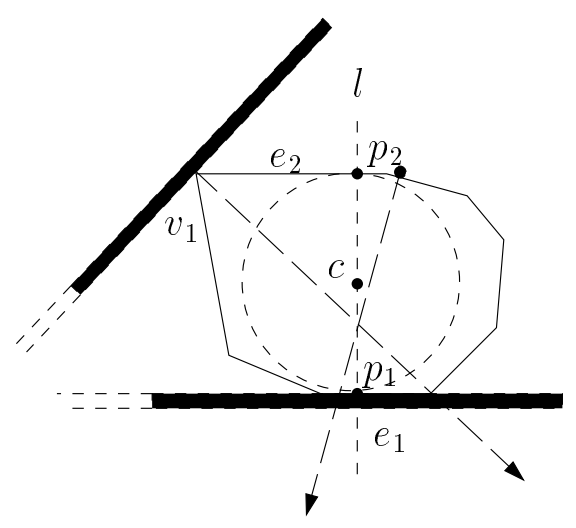

Figure 20: A polygon with one obtuse and one acute angle adjacent to edge $e_{2}$ as in Case 1 of the proof of Lemma 12.

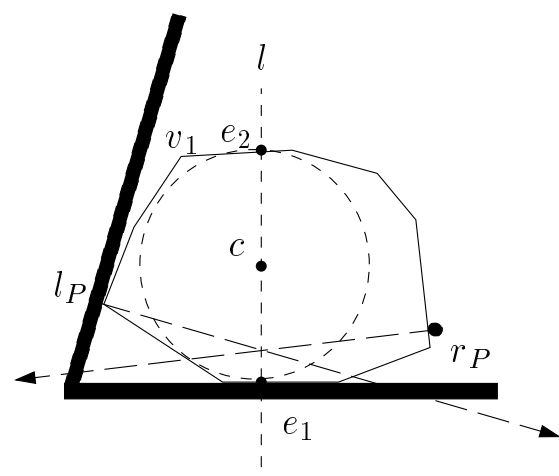

Figure 21: A polygon with two obtuse angles adjacent to edge $e_{2}$ as in Case 2 of the proof of Lemma 12.

Because of this orientation, we know that the intersection point of the contact normal to $a_{c}$ with $l$ will have a $y$-coordinate $y_{c}$ smaller than $y_{a_{2}}$.

Having fixed the point-contact, we now orient the angle-fixel such that the contact normal to $v_{1}$ intersects $l$ in the same point as the contact normal to $a_{c}$, which is always possible. The orientation of the angle fixel will never be 0 or $\frac{1}{2} \pi$, because $I_{p}$ does not contain the values $-\infty$ or $y_{a_{2}}$.

Case 2. Both edges adjacent to $e_{2}$ form an obtuse angle with $e_{2}$. See for example Figure 21.

Consider the $y$-coordinates of the highest left-most vertex $l_{P}$ of $P, y_{l}$ and of the lowest right-most point $r_{P}$ of $P, y_{r}$. Without loss of generality assume that $y_{l}>y_{r}$. We will now prove that we can find a form closure configuration by placing the edge-fixel to the left of $l$ and the point-fixel just above $r_{P}$.

Consider the edges with orientation in $I_{1}$ and their end-points. This is a chain of edges starting from $v_{1}$ and ending with vertex $l_{P}$. The chain will at least contain one vertex $\left(v_{1}\right)$. We now rotate the edge-fixel from angle 0 to $\frac{1}{2} \pi$. First 
the fixel will contact $v_{1}$ and an angle of $\frac{1}{2} \pi$ will result in a contact with $l_{P}$. During the rotation the edge will contact all vertices in the chain. If we look at the contact normals at the vertex of contact, during the rotation, we see that the intersection point with $l$ will move continuously from $y$-coordinate $-\infty$ to $y_{l_{P}}$. During the process, this point might have a $y$-coordinate above $y_{l_{P}}$, but we do not need these points.

We now have to find a point contact to the right of $l$ with orientation in $I_{2}$ and intersection point with $l$ somewhere below $y_{l_{P}}$. This is true for a point contact just above $r_{P}$. We can also find a form closure configuration in the case that $y_{l_{P}}=y_{r_{P}}$, but will not discuss this special case here.

A fixturability-proof can also be given for arbitrary non-convex polygons. In this case we can always construct a form-closure-configuration if we make the adjustable angle extremely small (such that both edges are nearly parallel). The construction though is not very stable and therefore rather impractical.

The time to compute a form-closure configuration depends on the time needed to compute the maximal inscribed circle of $P$. This is $O(n)$ [7].

\section{Modular Fixtures}

In this section we consider fixturing polygonal 2-dimensional objects with modular fixturing systems. We are given a rectangular flat surface into which circular holes have been drilled to form a regular square lattice. We assume that the size of the fixture table is large enough to place the fixture elements such that all possible form closure configurations can be accomplished. Grid points are hole centers while grid lines are the vertical and horizontal lines through the grid points. Unit distance is defined as the distance between two adjacent grid points on a single grid line. Let $P$ denote the given polygonal object with perimeter $p$ and diameter $d$, and let $P$ be defined by $n$ edges. In this section we will consider algorithms that compute all possible form closure configurations on a grid in a specific model. The fixels are assumed to be of zero size, thus they can be placed anywhere without intersections with other fixels or the object. (This restriction is not realistic but can often be enforced by taking the Minkovsky sum of the polygon and the fixel, thus reducing the fixel to a point. Also solutions can be checked afterwards to see whether they are actually realisable.) We will use the following fixture elements:

Locators. A locator must be placed at a grid point. It provides a point-contact with $P$. The locator has zero radius and the point of contact is a grid point.

Clamps. The fixed part of the clamp must be placed at a grid point. The extension of the clamp is assumed to be at most one grid unit along a grid line. In our model, where we assume that fixels have no size, this means that a clamp can be placed anywhere along a grid line. A clamp provides a point-contact with $P$ on a grid line. 
Edge-fixels. An edge-fixel is assumed to be attached to the lattice on one of the grid-lines. We normally put the fixel along the $x$-axis of our reference frame with $P$ in the positive $y$-half-plane. An edge-fixel provides an edge-edge contact with $P$.

Angle-fixels. An angle-fixel basically consists of two edge-fixels. One of the edge-fixels is placed horizontally along a grid-line, the other edge-fixel is attached to this fixel perpendicularly (Right-angle-fixel), such that it extends in the $y$-direction, or is attached by a joint, such that the angle with the horizontal edge-fixel is adjustable. The types of contacts with angle-fixels are discussed in the related subsections.

\subsection{Four Point-contacts}

We first discuss a number of different ways to fixture the polygon with four point-contacts.

\subsection{1 $3 \mathrm{~L} / 1 \mathrm{C}$}

Since achieving contact with four locators restricted to a grid is in general impossible, we need at least one fixel that takes care of the slack. So, the first model that we consider here is a model where we have three locators and one clamp $(3 \mathrm{~L} / 1 \mathrm{C})$. The existence of modular fixtures in this model was investigated by Zhuang et al. [31]. They proved a negative result (they identified a set of non-fixturable polygons). A complete algorithm to compute all possible form closure configurations was described by Brost and Goldberg [5]. We review both results briefly.

\section{Non-fixturability}

Intuitively one can see that a very small or long and skinny part might be unfixturable in the model, since three of the point-contacts (associated with the locators) must occur at a grid point. However, Zhuang et al. [31] proved the following theorem on convex polygons of arbitrary large diameter.

Theorem 13 [31] For any given diameter there exists a convex polygon of greater diameter that can not be fixtured on a grid with three locators and one clamp.

So, we can construct a convex part of arbitrary size that is unfixturable. The proof of the theorem consist of two main steps. First it is shown that for any given positive number $M$, a disk of radius $>M$ can be constructed that touches at most two grid points. The construction of this disk uses the finiteness of the set of all circles uniquely determined by all triplets of grid-points. It can then be shown that this disk can be transformed into a regular polygon while preserving this property. Since the part should contact three locators (placed at grid points) in order to obtain a form closure configuration, this regular polygon cannot be fixtured under the $3 L / 1 C$ model. 


\section{Algorithm to compute all form closures}

Brost and Goldberg [5] presented an algorithm that computes all possible form closure configurations in the $3 \mathrm{~L} / 1 \mathrm{C}$-model for polygonal objects. The algorithm is complete, since it outputs all possible form closure configurations. Furthermore, the algorithm can identify the optimal fixture according to an arbitrary quality metric. The main steps of the algorithm are the following.

1. For every combination of three (not all three identical) edges, the area swept out by the second edge by translating and rotating the first edge while maintaining contact with the origin of the grid is computed. This area is an annulus, of which only the part in the first quadrant has to be considered (to eliminate equivalent fixtures). Then for each grid point in this area, the possible positions for the third locator are in the intersection of the annulus defined by possible locations for the third edge with respect to the first and the annulus defined by the third edge with respect to the second locator position. This area can be refined by taking into account the angular limits of the part configurations that simultaneously contact the first and second locator.

2. Having found the triplets of locators and the corresponding contact edges, the set of consistent part configurations can be found by a so called configurationspace analysis. There may be two different poses of the part that permit simultaneous contact with the three chosen locators. In this case two candidate locator setups are generated.

3. For each of the generated locator-set-ups in the previous step, the region of possible clamp-positions is identified, using force-sphere analysis. The analysis identifies all possible positions where form-closure is obtained. The force-sphere represents all planar forces by direction ( $x$ and $y$ ) and moment components of a line of force exerted in the plane. A locator-clamp set-up should be able to resist all forces applied in the plane. The set of correct contact normals obtained with the force-sphere analysis, is then mapped back onto the part perimeter and intersected with the horizontal and vertical grid-lines to obtain the clamppositions that give a form closure configuration.

Brost and Goldberg prove the following theorem.

Theorem 14 [5] All configurations holding $P$ in form closure with three locators and one clamp can be computed in time $O\left(n^{4} d^{5}\right)$. The maximum number of possible fixtures is $O\left(n^{4} d^{5}\right)$.

\subsubsection{C or T-slots}

In order to be able to fixture more objects in a modular fixturing system with only four point-contacts than in the $3 L / 1 C$-model, we consider here a model with four clamps that turns out to be equivalent to the $T$-slot model considered in [31]. In this model we have four continuous degrees of freedom, wheras for a locator the number of possible positions is finite and discrete. We assume that 
the clamp can have an extension of at most one grid unit. Thus, the extension of a clamp can increase continuously from 0 to 1 grid-unit. So, in the $4 \mathrm{C}$-model, contacts that are made with the polygon can lie anywhere on a grid line. In this subsection we describe two classes of objects that were shown to be fixturable by Zhuang and Goldberg [31]. There is no algorithm yet that efficiently computes all form closure configurations in this model. It seems quite hard to do this, since we do not only have to deal with a discrete number of possible clamp positions, but also with four continuous degrees of freedom, namely the four extensions of the clamp plungers.

\section{Fixturable polygons}

Improving on a result of Mishra [20], Zhuang and Goldberg proved the following theorem. Remember that the distance between two adjacent grid points on a grid line is 1.

Theorem 15 [31] Let $P$ be a rectilinear polygon with all edges of length $>1$, then there always exists a fixture using at most 4 clamps.

The proof shows that a fixture always exists when $P$ is aligned with the axes of the lattice. Because of the restriction on the minimal length of the edges, we can always embed a unit-length square into $P$. By extending the four sides of this unit-square, we intersect the boundary of $P$ in eight points that are not vertices. It can then be shown that two sets of four of these points yield form closure for the part. The unit-square can be placed such that its vertices all lie on grid-points and thus the intended contact points are actually on grid-lines, which means that the four clamps can indeed be placed such that form closure is obtained.

Nguyen [22] showed how to find sets of 4 edge segments on an arbitrary polygon such that if we place a point contact somewhere on every of these four segments, the part is in form closure. Such segments are now called Nguyen segments. Zhuang and Goldberg also showed that a convex polygonal part is fixturable if there exists a set of at least three Nguyen segments of length $>\sqrt{2}$ [31]. Here we show another result, where existence of a fixture depends on the lengths of the edges of $P$ itself rather than the lengths of the Nguyen segments.

Theorem 16 Any polygon with all edges of length greater than $\sqrt{3}$ and without parallel edges is fixturable on a unit grid in the ${ }_{4} \mathrm{C}$ model.

Proof: Let $P$ be a polygon with all edges of length $>\sqrt{3}$ and without parallel edges. The maximal inscribed circle $M I C(P)$ of $P$, which is assumed to be centered at $c$, touches $P$ in three points. Assign the names $a_{1}, a_{2}$, and $a_{3}$ to these points in counterclockwise order such that $\angle a_{1} c a_{2} \leq \angle a_{2} c a_{3}, \angle a_{3} c a_{1}$. The vectors $\overrightarrow{a_{1}} c, \overrightarrow{a_{2}} c$, and $\overrightarrow{a_{3}} c$ positively span the plane. Moreover, $\angle a_{1} c a_{2} \leq 2 \pi / 3$.

Each of the three points $a_{1}, a_{2}$, and $a_{3}$ is either a point in the interior of an edge of $P$ or a concave vertex of $P$. In the case that a point $a_{i}(1 \leq i \leq 3)$ lies in the interior of an edge, a point contact at $a_{i}$ induces a half-plane constraint along the supporting line of $\overrightarrow{a_{i}} c$. In the case that $a_{i}$ is a concave vertex, a point 


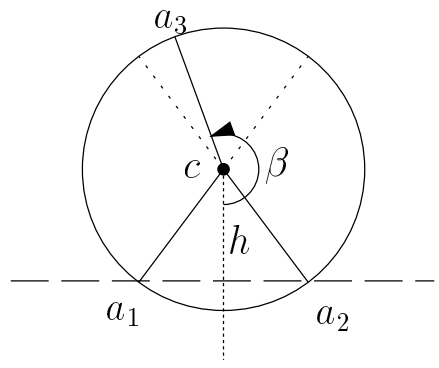

Figure 22: The points $a_{1}$ and $a_{2}$ are placed on horizontal grid line. The half-line $h$ bisects the angle between $c a_{1}$ and $c a_{2} ; \beta$ is the angle between $h$ and $c a_{3}$.

contact at $a_{i}$ induces two half-plane constraints whose conjunction implies the half-plane constraint along the supporting line of $\overrightarrow{a_{i} c}$. Moreover, the normals to the edges incident to $a_{i}$ positively span $\overrightarrow{a_{i} c}$. As a result, the composite constraint induced when $a_{i}$ is a concave vertex is stronger than the constraint induced when $a_{i}$ is a point in the interior of an edge.

As a first step, we put the two points $a_{1}$ and $a_{2}$ on a horizontal grid line and hold them with horizontal clamps. We assume that the constraints imposed at $a_{1}$ and $a_{2}$ are the weakest constraints, namely half-plane constraints along the supporting line of $\overrightarrow{a_{1} c}$ and $\overrightarrow{a_{2}} c$. Assume that $a_{3}$ lies above the horizontal grid line and let $h$ be the (vertical) half-line emanating from $c$ and bisecting $\angle a_{1} c a_{2}$. Let $\beta$ be the (counterclockwise) angle between $h$ and $c a_{3}$. Figure 22 shows the half-line $h$ and the angle $\beta$.

The fact that $\overrightarrow{a_{1}} c, \overrightarrow{a_{2}} c$, and $\overrightarrow{a_{3} c}$ positively span the plane and the bound $\angle a_{1} c a_{2} \leq 2 \pi / 3$ jointly imply that $2 \pi / 3 \leq \beta \leq 4 \pi / 3$. As a consequence, an edge tangent to $M I C(P)$ at $a_{3}$ cannot be too steep. In fact, the projection on a horizontal grid line of any such edge of length greater than $\sqrt{3}$ will have more than unit length.

Simply assuming that the constraint imposed by the contact at $a_{3}$ is a halfplane constraint would not give us form closure, as it will not exclude $c$ as a center of both clockwise and counterclockwise rotation. A more thorough analysis of the contact at $a_{3}$ is necessary to exclude $c$. We disinguish three cases for $a_{3}$ (see Figure 23).

$a_{3}$ is a concave vertex and neither of the edges incident to it is tangent to $M I C(P)$ :

Instead of a single half-plane constraint at $a_{3}$, the point contact at $a_{3}$ induces a wedge of half-plane constraints (see Section 3), leaving a half-wedge of centers of clockwise rotation and a half-wedge of centers of counterclockwise rotation. By the assumption that neither of the edges incident to the vertex $a_{3}$ is tangent to $M I C(P)$, the supporting line of $\overrightarrow{a_{3}} c$ lies in the interior of the wedge of constraints. As a result, $c$ is strictly in the interior of the wedge, excluding it as a center of both clockwise and counterclockwise rotation. Hence, $P$ is in form closure. By sliding $a_{1}$ and $a_{2}$ along the horizontal grid line we can put $a_{3}$ on 


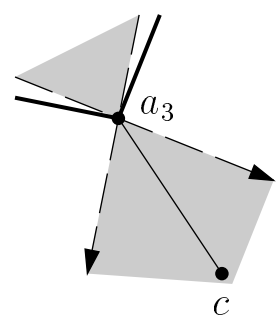

(a)

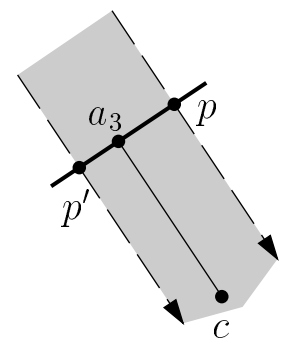

(b)

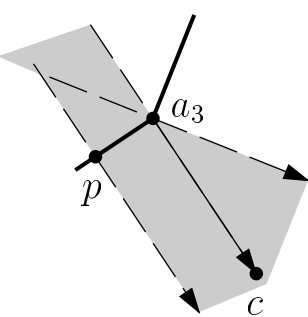

(c)

Figure 23: Three cases: (a) $c$ lies in the interior of the wedge induced by the point contact at $a_{3}$; (b) $c$ lies in the interior of the slab induced by the point contacts at $p$ and $p^{\prime}$; (c) $c$ lies in the interior of the union of the slab induced by the point contacts at $a_{3}$ and $p$ and the wedge induced by the point contact at $a_{3}$.

a vertical grid line, thereby establising that all contact points lie on grid lines. Note that in this case we need only three clamps.

\section{$a_{3}$ is a point in the interior of an edge:}

Since $a_{3}$ lies in the interior of an edge, we choose two points $p$ and $p^{\prime}$ on either side of $a_{3}$ on the same edge. Point contacts at $p$ and $p^{\prime}$ induce a slab constraint which has the supporting line of $\overrightarrow{a_{3}} c$ strictly in its interior. The slab constraint excludes $c$ as a center of clockwise and counterclockwise rotation. Hence, the point contacts at $a_{1}, a_{2}, p$, and $p^{\prime}$ hold $P$ in form closure. As each edge has length at least $\sqrt{3}$, we can choose $p$ and $p^{\prime}$ such that the projection of the segment $p p^{\prime}$ onto a horizontal grid line has unit length. By sliding $a_{1}$ and $a_{2}$ along the horizontal grid line we can put $p$ and $p^{\prime}$ on neighboring vertical grid lines, thereby establising that all contact points lie on grid lines.

$a_{3}$ is a concave vertex and (exactly) one of the edges incident to it is tangent to $M I C(P)$ :

Let $e$ be the edge tangent to $M I C(P)$ at $a_{3}$. We choose a point $p$ in the interior of the edge $p$. Point contacts at $p$ and $a_{3}$ induce a slab constraint and a wedge constraint which have the supporting line of $\overrightarrow{a_{3}} c$ as a common boundary. Careful analysis shows that $c$ lies strictly in the interior of the union of the slab and the wedge: $c$ lies to the right of the interior of the slab and to the left if the interior of the wedge, or vice versa. As a consequence, no rotation about $c$ is allowed. Hence, the point contacts at $a_{1}, a_{2}, a_{3}$, and $p$ hold $P$ in form closure. As each edge has length at least $\sqrt{3}$, we can choose $p$ such that the projection of the segment $a_{3} p$ onto a horizontal grid line has unit length. By sliding $a_{1}$ and $a_{2}$ along the horizontal grid line we can put $a_{3}$ and $p$ on neighboring vertical grid lines, thereby establising that all contact points lie on grid lines. 


\subsubsection{Vise}

Wallack and Canny [29] described an algorithm to compute all form closure configurations using a fixture vise. A vise consists of two modular fixturing tables, of which one can translate horizontally relative to the other table. Locators can be placed at the grid points of both tables. See for an example of a vise Figure 4. Here we have in addition to the discrete number of placements for the locators, only one continuous degree of freedom, namely the translation of one vise table in $x$-direction with respect to the second table. The two tables are also referred to as the left and right jaw. The fixture elements that are used are simply locators. If one of the locators is placed on one table and the other three on the other table, this model is similar to the $3 L / 1 C$-model (although there are some differences). A different case is encountered if two of the locators are placed on each fixture table. There are no known positive or negative results on existence of fixtures in this model.

\section{Algorithm to compute all form closures}

The algorithm of Wallack and Canny basically consists of two parts. In the first phase of the algorithm, all possible configurations of locators are generated, such that the locators are in contact with $P$. In the second phase of the algorithm, the corresponding orientation of the part is computed and then all configurations generated in the first phase are tested for form closure, using matrix computations (checking if the four contact points can resist arbitrary forces and torques). More specifically the first phase of the algorithm consist of three steps:

1. Enumerate all quadruple of edge-combinations of $P$, where every edge can occur more than once.

2. For each quadruple enumerate all different combinations of intended jawcontacts, i.e. for every edge specify if it will contact a locator on the right jaw or on the left jaw. Seven situations should be considered for every quadruple of edges (four where three edge segments contact the left jaw and three where pairs of edge-segments contact both jaws).

3. For each quadruple obtained in the previous step, compute fixture configurations providing simultaneous contact. This is done by choosing the first locator at some origin and then examining all possible positions for the second, third and fourth locator consecutively.

It should be clear that the last step is rather complex, regions that are swept out while maintaining contact with the already fixed locators should be described. In the paper, a description of these regions is computed exactly, using two different types of configurations, one where there are two locators on each table and the other one where there are three locators on one table and one on the other. Analysis of their algorithm gives the following theorem.

Theorem 17 [29] All configurations holding $P$ in form closure on a vise with 


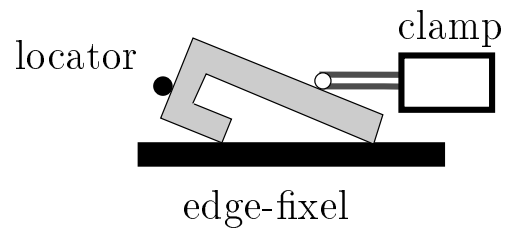

Figure 24: A form closure configuration with an edge-fixel, a clamp and a locator.

four locators can be computed in time $O\left(n^{4} d^{4} r^{2}\right)$, where $r$ is the largest range of distance between points on two edges. The maximum number of form closure configurations is also $O\left(n^{4} d^{4} r^{2}\right)$.

It should be noted that the algorithm uses this much time even when there are only a few resulting form closure configurations, since we only check for form closure after having generated all the possible contact positions of the locators.

\subsection{One Edge-fixel, a Locator and a Clamp}

In this section we consider an algorithm to compute all fixtures in a model with one horizontal edge-fixel, a locator and a clamp. The edge-fixel is assumed to be attached to the grid parallel to one of the horizontal grid-lines. We can distinguish two possibilities for placing the clamp. One, where the clamp is placed such that its extension is along a horizontal grid line (horizontal clamp) and the other case where the extension is along a vertical grid line (vertical clamp). Here, we will only discuss the algorithm for a horizontal clamp in detail. In addition we will briefly describe how to apply similar ideas to vertical clamps. We will also give a theorem about a class of rectilinear objects that can be fixtured on a modular grid.

\section{Algorithm to compute all form closures}

Since we know that at least four point contacts are necessary to fixture a polygon, we need to place $P$ always such that at least two point contacts are made between $P$ and the edge-fixel. The only assumption that we will make about the edge-fixel is that it is long enough, i.e. at least as long as the longest edge on the convex hull of $P$. $P$ will always be placed with an edge of the convex hull against the edge-fixel. Hence, we only have $O(n)$ different orientations for $P$, which makes the algorithm more efficient than algorithms for fixturing with four point-contacts.

To get a better understanding of the problem, we first give a naive (nonoutput sensitive) algorithm for computing all form closure configurations.

Consider the polygon $P$ shown bold in Figure 25 resting with some particular edge of its convex hull against the edge-fixel, the thick bar shown at the bottom. As $P$ may not be touching a grid-point on its boundary, we slide it, say to the left, until it does. Now we have a possible position for the locator. To compute all possible locator positions, it is sufficient to slide the polygon only one unit to the left and mark all grid-points encountered during the slide. Let $P=P(0)$ 


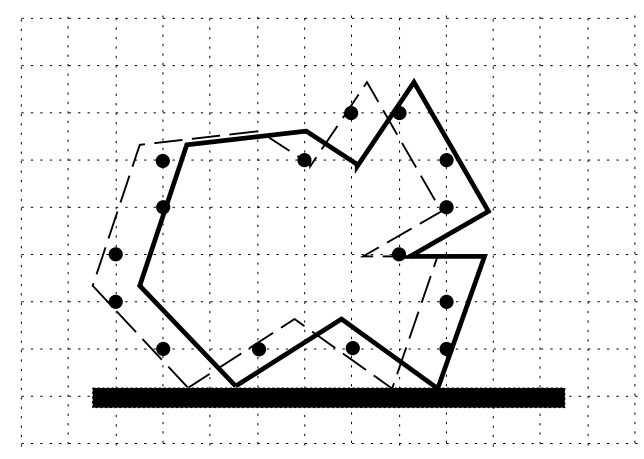

Figure 25: Sliding the polygon one unit along the edge-fixel to enumerate all feasible locator positions.

indicate the initial position of the polygon and $P(t)$, the polygon when shifted by distance $t$ to the left, $0 \leq t \leq 1$. We call $t$ the shift variable. A locator position $L$ exists only at certain discrete values of the shift variable; at each one it contacts a certain edge $e$ of $P$. Let us term each such contact as a locator-edge combination.

Clamp positions are those points on the boundary of $P$ intersected by the grid lines at some $P(t)$. We distinguish two cases: those points intersected by the horizontal grid lines are called horizontal clamp positions while the others are vertical clamp positions. Notice that since the shift is horizontal, horizontal clamp positions remain constant for all shift values and can be computed from the unshifted polygon. On the other hand, vertical clamp positions vary with the shift value. A vertical grid line might intersect several edges $e$ of $P$ during the shift resulting in (vertical) clamp-edge combinations.

There are at most $O(p)$ grid points (locator positions) generated by this process (a more precise bound is $O\left(\min \left(d^{2}, p\right)\right.$ ), where $d$ is the diameter of $P$ ). Furthermore, the total number of locator-edge and clamp-edge combinations is $O(n+p)$ and can be computed in $O(n+p)$ time.

A naive procedure to generate all valid fixtures is therefore to consider each locator-edge combination and clamp-edge combination pair, check if they simultaneously exist (w.r.t. the shift variable; this is necessary only for locators with vertical clamps) and if so, test if they achieve form-closure. Iterating over all convex hull edges against the edge-fixel gives a $\Theta\left(n(p+n)^{2}\right)$ algorithm.

\section{Horizontal clamp}

Instead of considering the placements of the locator and clamp (on the grid), we can also look at the possible contact normals associated with these grid points. We can then abstract from the grid and polygon and only have to look at directed lines. We assume that $P$ is placed with one of the edges of its convex hull $C H(P)$ against the edge-fixel. This edge-contact induces a slab as defined in Section 3. As mentioned before, horizontal clamp and locator contact points can be determined by intersecting the horizontal grid lines with the boundary 


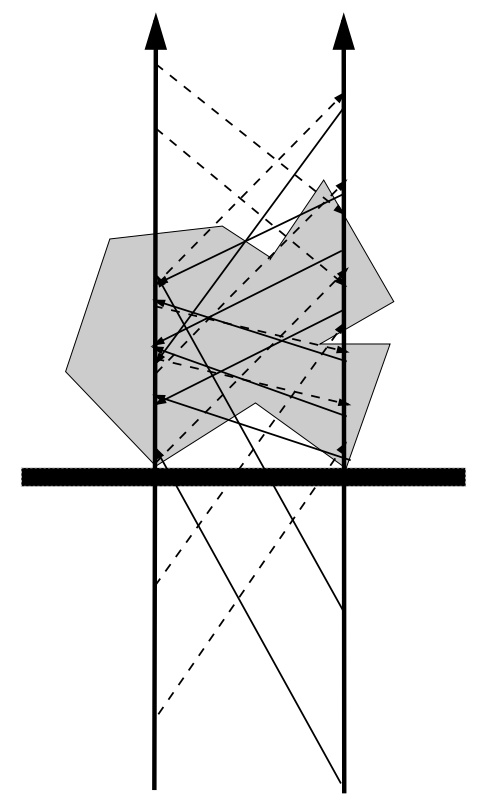

Figure 26: The directed line segments (directed lines representing half-plane constraints restricted to the slab formed by the edge-fixel at the bottom) resulting from the object in Figure 25. The dotted line segments (from left to right) correspond to the locators while the solid lines (from right to left) are from the horizontal clamps. 
of $P$. The contact points are always achievable, since we assume that the clamp can have a one grid-unit extension. Let us assume for now that the locator will be placed to the left of $P$ (meaning that by sliding $P$ from right to left, we will obtain a contact with it) and the clamp is placed to the right. We obtain two sets of lines. The clamp lines are the lines normal to the points of contact (as obtained by intersection of $P$ with the horizontal grid lines) directed into the object and having an orientation in the open interval $\left(\frac{1}{2} \pi, \frac{3}{2} \pi\right)$. This actually means that the lines cross the slab from right to left. There are $O(n+p)$ of these lines. The locator lines are the possible contact normals that cross the slab from left to right. The set of locator lines will will have $O(n+p)$ elements.

Thus we obtain two sets of directed lines defining half-plane constraints on $P$; one set of lines associated with possible clamp positions and one set of lines for the possible locator positions. We want to find all combinations of lines, one from each set, such that Conditions 1 and 2 in Lemma 4 are satisfied. Intersecting the directed (infinite) lines with the slab corresponding to the fixed convex hull edge of $P$, we obtain two sets of directed line segments. Given a locator line segment, we wish to detect, in an output-sensitive manner, all clamp line segments that (properly) intersect it and such that the vectors along the two segments and the upward vertical vector positively span $\mathbf{R}^{2}$. See Figure 26 . Formally, the basic query that we want to answer is:

QUERY Let $S$ be a set of $m$ directed line segments, each with the end-points along two vertical lines (the "slab"), and each with an orientation from the open interval $\left(-\frac{1}{2} \pi, \frac{1}{2} \pi\right)$. Store $S$ such that for a query segment $q$ also similarly anchored on the slab but with orientation in the interval $\left(\frac{1}{2} \pi, \frac{3}{2} \pi\right)$ one can report all segments $s \in S$ such that

1. the vectors along $s, q$ and the upward vertical vector positively span $\mathbf{R}^{2}$, and

2. $s$ and $q$ intersect strictly inside the slab.

Using a two-level partition tree, such queries can be solved in time $O\left(\log ^{2} m+\right.$ $k$ ), where $k$ is the number of reported segments (see [23] for details). Since we have to perform this query for all $O(n+p)$ clamp segments and $O(n)$ possible orientations of $P$, we obtain a total time of $O\left(n(n+p) \log ^{2}(n+p)+K\right)$, where $K$ is the number of form closure configurations. Actually in contrast to querying the clamp lines one at a time, we can process them all together and improve the time complexity to $O(n(n+p) \log (n+p)+K)$.

Theorem 18 [23] All configurations holding $P$ in form closure using one edgefixel, one locator and one horizontal clamp can be enumerated in time $O(n)(n+$ $\left.p) \log ^{2}(n+p)+K\right)$, where $K$ is the number of form closure configurations.

\section{Vertical clamp}

In the case of a vertical clamp we can apply a query algorithm that is similar to the one above. In this case, however, the place where the clamp will contact $P$ 's boundary depends on the extension of the clamp. As described in the naive 
algorithm above, we have to deal with an extra 'shift'-variable. The actual clamp-line for a specific clamp position depends on this variable. Locator lines exist only at one time during the shift (when an edge of the polygon shifts over the corresponding grid-point) In order to solve this problem, we can add an extra dimension to the query problem, representing the shift. In this query structure a locator line is a segment inside a block and clamp lines are represented by parallelograms inside a block. Again, we query the structure with every parallelogram. Because of the three-dimensional nature of this problem this leads to the following result.

Theorem 19 [23] All configurations holding $P$ in form closure using one edgefixel, one locator and one vertical clamp can be enumerated in time $O(n)+$ $\left.p)^{\frac{4}{3}+\epsilon}+K\right)$, where $K$ is the number of form closure configurations.

\section{Fixturable polygons}

We can prove that a subset of rectilinear polygons is fixturable under the edgelocator-clamp (ELC)-model. The result is comparable to [31]. Specifically, we are interested in simple polygons that satisfy the following conditions.

1. The edges of the polygon are parallel or perpendicular to each other (rectilinear polygons).

2. The convex hull of the polygon is not a rectangle.

3. Each edge is of length at least $\delta$ grid units.

We term such a class of polygons as $\delta$-long rectilinear polygons. By Condition 2, they are necessarily non-convex. The convex hull of such a polygon has at least one edge that is neither parallel nor perpendicular to the edges of the polygon. Such convex hull edges are termed skew edges.

Theorem $202+\sqrt{2}$-long rectilinear polygons are fixturable with an edge-fixel, a locator and a clamp.

Proof: We can show that for every configuration of a $\delta$-long rectilinear polygon $P$ in which a skew edge rests against the edge-fixel, $P$ can be set into formclosure by a locator and a clamp if $\delta \geq 2+\sqrt{2}$. In this proof we will use the name of a segment to denote the length of the segment as well. Consider a $\delta$-long rectilinear polygon placed with a skew edge against the edge-fixel as shown in Figure 27. Consider the two edges $e_{i}$ and $e_{j}$ of $P$ joining the two points of contact, $e_{i}$ extending to the right from the leftmost contact vertex and $e_{j}$ extending to the left of the rightmost contact vertex. Let $T$ be the triangle defined by the skew edge and the supporting lines of $e_{i}$ and $e_{j}$. Let $a$ and $b$ be the triangle edges along $e_{i}$ and $e_{j}$ respectively. Since $e_{i}$ and $e_{j}$ do not intersect each other, at least one of them will be fully contained in $a$ or $b$. Observe that this will be the least steep edge of the two. Without loss of generality let us assume that the leftmost edge of $e_{i}$ and $e_{j}$ is fully contained in $T$. Thus, $a \geq \delta$. 


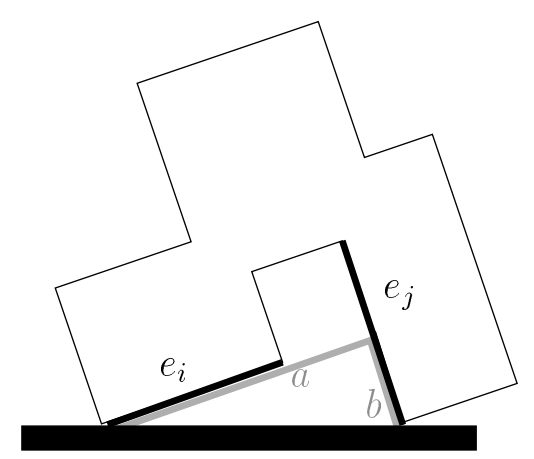

Figure 27: Proving a class of rectilinear polygons as fixturable.

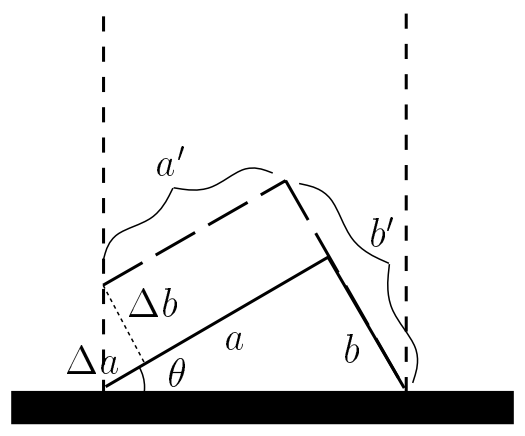

Figure 28: Extending $a$ and $b$ such that they are equally long inside the slab.

Let $\theta=\tan ^{-1} b / a \in\left(0, \frac{1}{4} \pi\right]$. Orient the edges of the polygon in counterclockwise order. Then in this particular configuration, the orientations of the edges of the polygon fall into four categories:

$$
\theta, \pi / 2+\theta, \pi+\theta, 3 \pi / 2+\theta
$$

Further, observe that, for form-closure purposes, the only locator/clamp contact points possible are along edges with orientations

$$
\pi / 2+\theta, \pi+\theta
$$

the locator being on an edge from one orientation class and the clamp contact point on an edge from the other. Let us call these the principle orientations. This is only a necessary condition. For sufficiency we also require that the two directed lines representing the half-plane constraints intersect inside the slab defined by the points of contact with the edge-fixel.

To ensure the second condition, we can project the edges $a$ and $b$ onto the edges of $P$ with the principal orientations, one point-contact placed in the projection of $a$ and one in the projection of $b$ will give a form-closure configuration. 
However, since we do not know anything about the length of $b$ and its projection, we do not know if we can place a clamp or locator here. This is why we first extend edge $b$, obtaining a new edge $b^{\prime}=b+\Delta b$ and move $a$ such that it extends from $b^{\prime}$. This gives us two new edges, $a^{\prime}=a-\Delta a$ and $b^{\prime}$ inside the slab, such that their projection onto the edges with principal orientations will give us positions for a form closure configuration. See Figure 28. In order to say something about the edge-length $\delta$ of the polygon, such that we can always place a locator in the projection of $a^{\prime}$ and a clamp in the projection of $b^{\prime}$, we will compute the length of $a^{\prime}$ and $b^{\prime}$ if $a^{\prime}=b^{\prime}$, because we would like to have both projections as long as possible. We can show that

$$
\Delta a=\Delta b \tan \theta
$$

and

$$
b=a \tan \theta .
$$

Thus,

$$
b+\Delta b=a-\Delta b \tan \theta,
$$

which gives us

$$
\Delta b=(a-a \tan \theta) /(1+\tan \theta) .
$$

Substitution of (2) and (4) in $b^{\prime}=b+\Delta b$ gives us

$$
\begin{aligned}
b^{\prime} & =a\left(\tan ^{2} \theta+1\right) /(\tan \theta+1) \\
& >\delta\left(\tan ^{2} \theta+1\right) /(\tan \theta+1)
\end{aligned}
$$

The function $f(\theta)=\left(\tan ^{2} \theta+1\right) /(\tan \theta+1)$ has its minimum on the interval $\left(0, \frac{1}{4} \pi\right]$ at $\theta=\frac{\pi}{8}, f\left(\frac{\pi}{8}\right)=2 \sqrt{2}-2$. This means that

$$
b^{\prime}=a^{\prime}>\delta(2 \sqrt{2}-2) .
$$

However, in the rest of the proof it seems better to extend $b$ until $a^{\prime}=b^{\prime}$ if $a^{\prime}=b^{\prime}<e_{j}$, or until its length equals the length of $e_{j}$.

Consider the outermost projections of $a^{\prime}$ and $b^{\prime}$ onto $P$. By this we mean the edges with principal orientation inside the slabs defined by $a^{\prime}$ and $b^{\prime}$, starting from the edge that intersects the first (lower) slabline and whose intersection point with the slabline has the highest $y$-coordinate. Then we walk along the polygon starting towards the interior of the slab until we meet the second slabline. If we meet the first slabline again, we will forget about the edges that we have visited and start all over again at the next intersection point with the first slabline. All edges with the correct orientation that we visit during our walk towards the second slabline are the edges that we will consider. See for an example Figure 29. For $s l a b\left(b^{\prime}\right)$, we know that we will always reach the second slabline, since edge $e_{j}$ intersects both slablines. So there exists a point of $P$ to the right of or on the second slabline. For $\operatorname{slab}\left(a^{\prime}\right)$, we know it, because there is a part of the polygon at both sides of the slab (edge $e_{j}$ is to the right of the slab and the edge extending to the left from the leftmost contact vertex is to the left of the slab, since $\operatorname{slab}\left(a^{\prime}\right)$ is contained in $\left.\operatorname{slab}(a)\right)$, so we know that there 


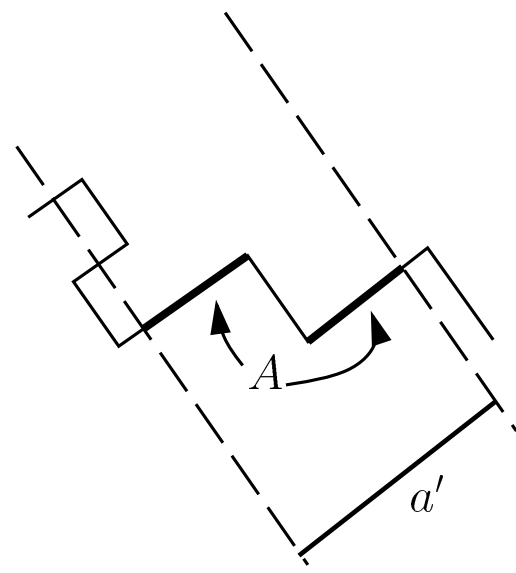

Figure 29: Projection of $a^{\prime}$ onto the principal edges of $P$.

should be edges that connect the parts to the left and to the right of the slab that will intersect the slab. Let the obtained sets of projected edges be $A$ and $B$. If both sets contain one edge of length at least $\sqrt{2}$, then we can obtain form closure. This is so, because the edge from $A$ will intersect a vertical grid line and the edge from $B$ a horizontal grid line. We can slide $P$ to the right until the edge from $B$ encounters a grid-point. Place the locator there. Because of the minimal edge-length of $\sqrt{2}$ a vertical grid line is still intersected after the shift of $P$ against the locator. Thus this vertical grid line gives the clamp position.

In order to have a segment in $A$ of length at least $\sqrt{2}$, we should have that the width of the slab (which is equal to $a^{\prime}$ and $b^{\prime}$ ) should be at least $2 \sqrt{2}$. According to Equation (7), in that case $\delta \geq 2+\sqrt{2}$. Then, if $A$ contains one single edge it is clear that this edge has length at least $2 \sqrt{2}$ (the width of the slab). If $A$ contains two edges, the length of one of the edges is of length $\geq 2 \sqrt{2} / 2$ which is at least $\sqrt{2}$. If $A$ contains three or more edges, at least one of them is fully contained in the slab, (observe that only two of the edges are cut by a slabline according to our definition of outermost projection) meaning that its length is at least $\delta$ and thus larger than $2+\sqrt{2}$. The same arguments hold for the projection $B$ if we extended $b$ such that $a^{\prime}=b^{\prime}$. In the other case where we extended $b$ until the length of $e_{j}$, we know that the width of $\operatorname{slab}\left(b^{\prime}\right)$ is $>\delta$, since this is the minimum edge length for $e_{j}$. Thus we can apply similar arguments as above and see that there will always be an edge of length at least $\sqrt{2}$ in the projection $B$. Thus we have shown that we can fixture $\delta$-long polygons if $\delta>2+\sqrt{2}$.

Such a fixture can be computed in $O(n)$ time. Also note that as a consequence of Theorem 20, in the absence of a grid any rectilinear polygon for which the convex hull is not a rectangle can be fixtured with an edge and two points. This extends Theorem 5 in Section 5 . 


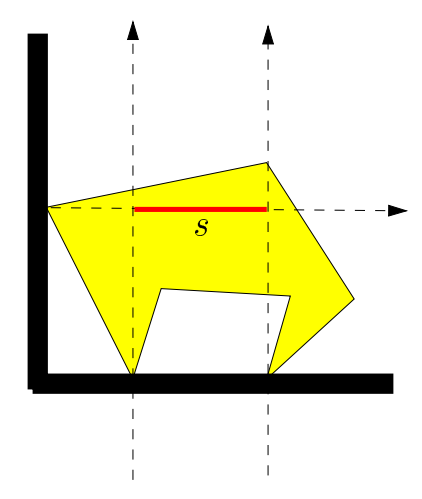

Figure 30: The segment $s$ that should be intersected by the contact normal to the clamp contact when $P$ is placed against a right-angle fixel.

\subsection{One Angle-fixel and a Clamp}

Finally we discuss modular fixtures using an angle-fixel and one clamp. The three types of angle-fixels that we will consider are a so-called right-angle fixel, a fixed-angle fixel, where the angle is not $\frac{1}{2} \pi$ and the adjustable angle fixel. No results are known about what objects can be fixtured in this model. The algorithms described here are quite straightforward and follow directly from the geometric interpretation of the contacts.

\section{Right angle-fixel and a clamp}

In order to obtain a form closure configuration $P$ will always be placed with one of the edges of $C H(P)$ against one of the edges of the fixel. Assume this to be the horizontal edge. $P$ is then slided along the horizontal edge, until it hits the second, vertical edge of the fixel. Usually this will yield a contact of a vertex of $P$ with the vertical edge. Having done this, the orientation and position of $P$ are fixed. For a fixed orientation of $P$, we can compute the intersection between the slab defined by the edge-contact with the horizontal edge of the angle-fixel and the contact normal to the point-contact with the vertical edge of the fixel (Lemma 6). The angle-fixel can be placed to the left or to the right of $P$. The computation of all form closure configurations is similar for both cases. Let us assume here that the right-angle fixel is placed to the left of the polygon as in Figure 30. The intersection of the contact normals give us a horizontal segment $s$. See Figure 30. The possible contact points for the clamp can be obtained by intersection of the boundary of $P$ with the horizontal and vertical grid lines. This gives us $O(n+p)$ possible contact points and their corresponding contact normals. For every element in this set we now have to check if they have an orientation in the interval $\left(\pi, 1 \frac{1}{2} \pi\right)$ and if they intersect $s$. Since we have to do this for every orientation of $P$, we get the following result.

Theorem 21 All configurations holding $P$ in form closure with a right-angle fixel and a clamp can be enumerated in time $O(n(n+p))$. 


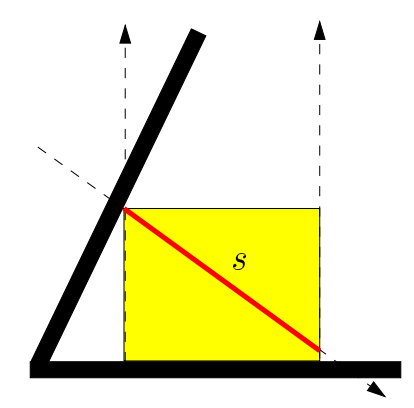

Figure 31: The segment $s$ that should be intersected by the contact normal to the clamp contact when $P$ is placed against a fixed-angle fixel.

Note that when $P$ also touches the second, vertical, edge of the fixel with an edge of its convex hull, instead of a segment $s$, we obtain a rectangle that should be intersected by the contact normal of the clamp contact. This does not increase the time bound.

\section{Fixed angle-fixel and a clamp}

The algorithm as described above for the case of a right-angle fixel can equally be applied to the case of an arbitrary fixed-angle fixel. The only things that we have to change are the check for the orientation of the contact normal to the clamp, if the angle of the fixel is $\alpha$, the angle of the contact normal to the second, non-horizontal edge will have direction $\left(1 \frac{1}{2} \pi+\alpha\right)$. This means that the orientation of the contact normals that will provide form closure must be somewhere in the interval $\left(\pi+\alpha, 1 \frac{1}{2} \pi\right)$.

Theorem 22 All configurations holding $P$ in form closure with one fixed-angle fixel and a clamp, can be computed in time $O(n(n+p))$.

\section{Adjustable angle-fixel and a clamp}

If the angle of the angle-fixel is adjustable, the number of form closure configurations can be infinite, it might be possible to generate intervals of angles for the fixel together with a finite number of possible clamp positions that will provide form closure. No such algorithm currently exists. Another possibility is to restrict the form closure configurations to those configurations where both edges of the fixel are in contact with an edge of $C H(P)$. In this way though an existing solution might be overlooked, for example for a rectangle we will not find a form closure configuration, although one exists.

\section{Extensions and Open Problems}

The algorithms described in this paper only give results for a basic situation (fixels of zero size, no quality metrics applied, only two-dimensional polygonal objects). Some of the algorithms can be extended or changed such that additional cases can be dealt with as well. Here we give a short overview of a 


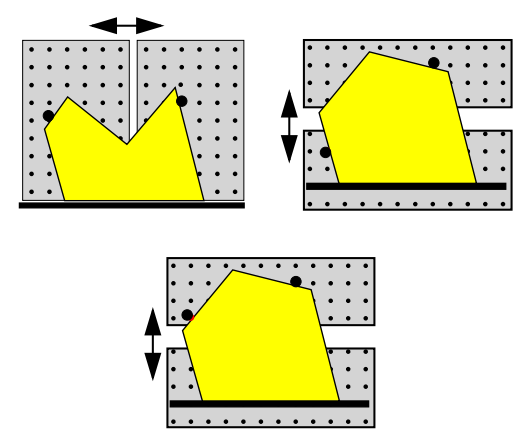

Figure 32: Three different models for a vise combined with an edge-fixel.

number of known extensions.

\section{Computation of Nguyen regions}

Nguyen [22] introduced an algorithm to compute Nguyen regions of an object in a model with four point contacts. Nguyen regions define possible placements of the point contacts, such that form closure is obtained if we place at least one contact in every region. Since the Nguyen regions are independent of a grid, we can usually find more efficient algorithms to compute these regions than to compute all possible fixtures on a grid. Nguyen described an $O\left(n^{4}\right)$ algorithm to compute al these combinations of regions. In the case of a right-angled fixel and a point contact, all regions where we can place the point contact for all orientations of $P$ can be computed in time $O\left(n \log ^{3} n+K\right)$ time, where $K$ is the number of resulting regions. This can be achieved by adding an extra level in the partition tree. In the case of one edge and two point contacts we currently only have efficient algorithms for rectilinear polygons and c-oriented polygons.

\section{Fixels with size $>0$}

Up to now we assumed the fixels to have 0 size. This is highly unrealistic. To deal with locators of non-zero size one can compute the Minkovsky sum of the polygon with the locator. This blows up the polygon and reduces the locator to a point. For the clamps we can either test for intersecting afterwards or use similar techniques. Finally, the size of the edge-fixels never causes a problem (it only shifts the polygon with respect to the grid). For example, the algorithm of Brost and Goldberg [5] thus needs time $O\left(n^{5} d^{5}\right)$. The time complexity of the vise-algorithm of Wallack and Canny [29] (Theorem 17) does not change. The Minkovsky difference can also be embedded in the algorithm for one edge-fixel, a locator and a clamp [23] (Theorems 18 and 19), The time for a vertical clamp does not change (Theorem 19), for a horizontal clamp we get an $O\left(n(n+p) \log ^{3}(n+p)+K\right)$ algorithm.

\section{Vise with wall}

Wallack and Canny [29] considered a vise with four locators, but what happens if we replace two of the locators by an edge-fixel? We can distinguish three 
cases. See Figure 32. In the first case the edge-fixel is placed in horizontal direction (the direction of the translation of one of the two tables of the vise). In addition one locator is placed on each of the tables of the vise. We can generate all form-closure configurations with the output-sensitive algorithm for an edge-fixel, a locator and a horizontal clamp (Theorem 18), since we can view the locator on the moving table as a horizontal clamp. If the edge-fixel is placed perpendicular to the direction of movement of the vise, we can obtain form closure for two types of placements of the locators. In the first case the locators are both on different tables. This case is similar to a model with one edge-contact, one locator and one vertical clamp and we can use the algorithm for this model to compute all form closure configurations (Theorem 19). In the other case, the two locators are both placed on the top table (the moving table). It is an open problem how to compute all form closure configurations in an output-sensitive way in this case.

\section{Curved edges}

Wallack and Canny [30] also presented an algorithm to compute form closure configurations on a vise, where the objects to be fixtured are so called generalized polygons. These are two-dimensional objects with a boundary composed of linear edges and circular arcs. It is unclear how to generalize the other algorithms in this paper to polygons with curved edges.

\section{Three dimensional fixturing}

Markenscoff et al. showed that for three-dimensional polyhedra, seven frictionless point contacts are necessary and sufficient to fixture the part [19]. In addition Wagner et al. [28] proposed a three-dimensional modular fixturing system using seven struts that are mounted to the walls of a rectangular four-sided frame on which mounting-sockets are arranged in a regular grid. They also presented an algorithm that enumerates all fixtures for a polyhedron, whose pose is given. All possible seven-membered sets of simultaneously contacting strut-positions are enumerated and tested for form closure, leading to a $O\left(t^{7}\right)$ algorithm, where $t$ is the number of lattice sites in the projection of the faces of the polyhedron on the fixture frame. Clearly, this bound is quite high and can possibly be improved. An open question is yet how to determine all possible poses of the polyhedron. It would be nice if there was a graphical representation of possible motions in 3-D similar to the 2-D representation of Reuleaux [25], since this would probably help in finding output-sensitive algorithms.

\section{Quality metrics}

After we have generated a list of all possible form closure configurations for a given part, we would like to rank them according to some quality metric. Several quality measures have been proposed in literature based on the smallest contact force necessary to resist applied forces $[9,18]$. One such measure is the radius of the largest sphere that can be embedded inside the wrench convex $[12,17]$. Brost and Goldberg [5] describe a quality metric that is used to rank the fixtures obtained by their algorithm for three locators and a clamp. The metric allows the user to specify a list of expected forces on the part. The 
quality metric scores each fixture by estimating the maximum contact reaction force required to resist the list of expected applied forces. However, any suitable quality metric can be applied, since ranking the fixtures is done as an extra final step of their algorithm. It would be nice if, rather than checking all solutions afterwards, one could incorporate the metric in the algorithm itself. Thus, hopefully, reducing the running time.

As can be seen from this paper much progress has been made in fixture design over the past few years but many questions are still open. For example, only partial results are known about which polygons can be fixtured in which model (either with or without a grid). Also many of the algorithms for modular fixturing seem to be far from optimal. Finally, the extensions to curved objects, three-dimensional fixtures and incorporation of the quality metric are far from being solved.

\section{References}

[1] H. Asada and A. B. By. Kinematic analysis of workpart fixturing for flexible assembly with automatically reconfigurable fixtures. IEEE Journal of Robotics and Automation, RA-1(2):86-94, June 1985.

[2] J. J. Bausch and K. Youcef-Toumi. Kinematic methods for automated fixture reconfiguration planning. In International Conference on Robotics and Automation, pages 1396-1401. IEEE, May 1990.

[3] A. Blake and M. Taylor. Planning planar grasps of smooth contours. In International Conference on Robotics and Automation, pages 834-839. IEEE, May 1993.

[4] W. Boyes, editor. Handbook of Jig and Fixture Design, 2nd Edition. Society of Manufacturing Engineers, 1989.

[5] R. C. Brost and K. Y. Goldberg. A complete algorithm for synthesizing modular fixtures for polygonal parts. In International Conference on Robotics and Automation, pages 535-549. IEEE, May 1994.

[6] R. C. Brost and M. T. Mason. Graphical analysis of planar rigidbody dynamics with multiple frictional contacts. In H. Miura and S. Arimoto, editors, Robotics Research: The Fifth International Symposium, pages 293300. MIT Press, 1990.

[7] Francis Chin, Jack Snoeyink, and Cao-An Wang. Finding the medial axis of a simple polygon in linear time. In Proc. 6th Annu. Internat. Sympos. Algorithms Comput. (ISAAC 95), volume 1004 of Lecture Notes in Computer Science, pages 382-391. Springer-Verlag, 1995.

[8] Y-C. Chou, V. Chandru, and M. M. Barash. A mathematical approach to automatic configuration of machining fixtures: Analysis and synthesis. Journal of Engineering for Industry, 111:299-306, November 1989. 
[9] M. R. Cutkosky. Grasping and fine manipulation for automated manufacturing. PhD thesis, Carnegie-Mellon University, Dep. Mech. Eng., Jan 1985.

[10] J. Czyzowicz, I. Stojmenovic, and J. Urrutia. Immobilizing a polytope. In Lecture Notes in Computer Science, volume 519, pages 214-227. 1991.

[11] B. Faverjon and J. Ponce. On computing two-finger force-closure grasps of curved $2 \mathrm{~d}$ objects. In International Conference on Robotics and Automation. IEEE, May 1991.

[12] C. Ferrari and J. Canny. Planning optimal grasps. In International Conference on Robotics and Automation, pages 2290-2295. IEEE, May 1992.

[13] M. V. Gandhi and B. S. Thompson. Automated design of modular fixtures for flexible manufacturing systems. Journal of Manufacturing Systems, $5(4): 243-252,1986$.

[14] E. G. Hoffman. Modular Fixturing. Manufacturing Technology Press, Lake Geneva, Wisconsin, 1987.

[15] M. E. Houle and G. T. Toussaint. Computing the width of a set. IEEE Trans. Pattern Anal. Mach. Intell., PAMI-10(5):761-765, 1988.

[16] D. G. Kirkpatrick, B. Mishra, and C-K Yap. Quantitative steinitz's theorems with applications to multifingered grasping. In ACM Annual Symposium on Theory of Computing, pages 341-351, 1990.

[17] Z. Li and S. S. Sastry. Task-oriented optimal grasping by multifingered robot hands. IEEE Transactions on Robotics and Automation, RA-4(1):3243, 1988 .

[18] X. Markenscoff, L. Ni, and C.H. Papadimitriou. The geometry of grasping. International Journal of Robotics Research, 9(1):61-74, 1990.

[19] B. Mishra. Workholding: Analysis and planning. In International Conference on Intelligent Robots and Systems, pages 53-56, July 1991.

[20] B. Mishra, J. T. Schwartz, and M. Sharir. On the existence and synthesis of multifinger positive grips. Algorithmica, 2(4):641-558, 1987.

[21] V-D. Nguyen. Constructing force-closure grasps. International Journal of Robotics Research, 7(3):3-16, 1988.

[22] M. Overmars, A. Rao, O. Schwarzkopf, and C. Wentink. Immobilizing polygons against a wall. In ACM Symposium on Computational Geometry, pages 29-38, June 1995.

[23] J. Ponce, S. Sullivan, J-D. Boissonnat, and J-P. Merlet. On characterizing and computing three- and four-finger force-closure grasps of polyhedral objects. In IEEE Conference on Robotics and Automation, pages 821-827, May 1993. 
[24] F. Reuleaux. The Kinematics of Machinery. Macmilly and Company, 1876. Republished by Dover in 1963.

[25] E. Rimon and J. Burdick. On force and form closure for multiple finger grasps. In IEEE Conference on Robotics and Automation, 1996.

[26] J. C. Trinkle. On the stability and instantaneous velocity of grasped frictionless objects. IEEE Transactions on Robotics and Automation, $8(5): 560-572,1992$.

[27] R. Wagner, Y. Zhuang, and K. Goldberg. Fixturing faceted parts with seven modular struts. In IEEE International Symposium on Assembly and Task Planning, pages 133-139, August 1995.

[28] A. Wallack and J. Canny. Planning for modular and hybrid fixtures. In IEEE Conference on Robotics and Automation, pages 520-527, May 1994.

[29] A. Wallack and J. Canny. Modular fixture design for generalized polyhedra. In IEEE Conference on Robotics and Automation, April 1996.

[30] Y. Zhuang and K. Goldberg. On the existence of solutions in modular fixturing. International Journal of Robotics Research, 1996. to appear. 